\title{
Comparison between two-fluid model simulations and particle image analysis \& velocimetry (PIV) results for a two-dimensional gas-solid fluidized bed
}

\author{
F. Hernández-Jiménez*, S. Sánchez-Delgado, A. Gómez-García, A. Acosta-Iborra \\ Departamento de Ingeniería Térmica y de Fluidos, Universidad Carlos III de Madrid, ISE Research Group, Avda. Universidad, 30,28911 Leganés, Madrid, Spain
}

\begin{abstract}
A B S T R A C T
This work compares simulation and experimental results of the hydrodynamics of a two dimensional, bubbling air fluidized bed. The simulation in this study has been conducted using an Eulerian Eulerian two fluid approach based on two different and well known closure models for the gas particle interaction: the drag models due to Gidaspow and Syamlal \& O'Brien. The experimental results have been obtained by means of Digital Image Analysis (DIA) and Particle Image Velocimetry (PIV) techniques applied on a real bubbling fluidized bed of $0.005 \mathrm{~m}$ thickness to ensure its two dimensional behaviour. Several results have been obtained in this work from both simulation and experiments and mutually compared. Previous studies in literature devoted to the comparison between two fluid models and experiments are usually focused on bubble behaviour (i.e. bubble velocity and diameter) and dense phase distribution. However, the present work examines and compares not only the bubble hydrodynamics and dense phase probability within the bed, but also the time averaged vertical and horizontal component of the dense phase velocity, the air throughflow and the instantaneous interaction between bubbles and dense phase. Besides, quantitative comparison of the time averaged dense phase probability as well as the velocity profiles at various distances from the distributor has been undertaken in this study by means of the definition of a discrepancy factor, which accounts for the quadratic difference between simulation and experiments The resulting comparison shows and acceptable resemblance between simulation and experiments for dense phase probability, and good agreement for bubble diameter and velocity in two dimensional beds, which is in harmony with other previous studies. However, regarding the time averaged velocity of the dense phase, the present study clearly reveals that simulation and experiments only agree qualitatively in the two dimensional bed tested, the vertical component of the simulated dense phase velocity being nearly an order of magnitude larger than the one obtained from the PIV experiments. This discrepancy increases with the height above the distributor of the two dimensional bed, and it is even larger for the horizontal component of the time averaged dense phase velocity. In other words, the results presented in this work indicate that the fine agreement commonly encountered between simulated and real beds on bubble hydrodynamics is not a sufficient condition to ensure that the dense phase velocity obtained with two fluid models is similar to that from experimental measurements on two dimensional beds.
\end{abstract}

\section{Introduction}

Large scale gas fluidized beds are used in many branches of engineering and science, including catalytic cracking, controlled combustion and gasification, and a variety of chemical operations (Kunii and Levenspiel, 1991; Basu, 2006; Yang, 2003). Current research on gas fluidized beds requires an ever increasing knowl edge of the hydrodynamics of the gas and dense phase, the simulation being a key part in the understanding, design and optimisation of such systems.

In order to simulate large scale gas fluidized beds, it is essential to use efficient numerical algorithms that combine reliability with not very long computing times. Four main approaches can be found in literature for the simulation of fluidised beds depending on the level of detail and resolution required (Grace and Taghipour, 2004; Van der Hoef et al., 2005). The most detailed approach is the direct simulation of the fluid flow surrounding solid particles and the interaction between them. For such approach lattice Boltzmann methods are normally used (see, for example, Ladd and Verberg, 2001). Certainly, the 
direct simulation of gas fluidized beds demands large computa tional resources, surpassing the current hardware capabilities unless a relatively small number of particles are to be simulated. A second approach in the simulation of fluidized beds is the direct particle or discrete element modelling, which is based on the Lagrangian simulation of each particle trajectory coupled with the Eulerian simulation of the bulk gas flow, the solid gas interaction being computed through semi empirical losure models to dimin ish the level of detail required in the solution of the gas phase (Deen et al., 2007). Although very promising, this Lagrangian Eulerian approach is still computationally intensive, leading to inadmissible memory needs and computing times when simulat ing the huge amount of particles normally encountered in medium or large scale fluidized beds. The level of resolution in particle motion can be reduced by computing only the bulk velocity behaviour of particles and not their individual trajec tories. This is the essence of a third approach known as Eulerian Eulerian, or CFD, two fluid modelling of fluidized beds (Gidaspow, 1994; Van Wachem and Almstedt, 2003). In this approach the gas phase and the particle or solids phase are treated as two inter penetrating and continuum media in an Eulerian framework using the conservation equation of fluids. As in the case of the Lagrangian Eulerian approach, the two fluid simulation of flui dized beds requires the use of closure models for the gas solids interaction. However, the lack of detailed description of particle motion in two fluid simulation necessitates also of the inclusion of sophisticated particle particle interaction models, which are normally based on empirical and theoretical relations linked to the kinetic theory of gases through the concept of granular temperature (Gidaspow, 1994). A fourth approach can be followed using Bubble discrete models and phenomenological methods, in which only the most relevant macroscopic characteristics of the bed are reproduced, e.g. gross bubble behaviour and general motion of the emulsion phase (see, for example, Bokkers et al., 2006). As this approach is based on simplified models and phenomenological correlations, it is the less computationally expensive and, therefore, it is especially suitable for the simulation and optimisation of large scale fluidized beds supporting chemical reactions such as combustion or gasification.

The Eulerian Eulerian two fluid approach is by far the method most encountered in current simulation of fluidized beds. This is perhaps due to its compromise between computational cost, level of detail provided, and potential of applicability. As a conse quence, there is an increasing need of verification and validation of the closure models utilised in the two fluid simulation of gas fluidized beds in different operative conditions and applications (see, for instance, Peirano et al., 2001; McKeen and Pugsley, 2003; Patil et al., 2005; Taghipour et al., 2005; Li et al., 2009). However, as some authors have pointed out (Grace and Taghipour, 2004), this verification and validation should be interpreted and extra polated with caution due to the complex nature of fluidized beds.

The majority of the works comparing two fluid models and experiment results are primarily focused on the behaviour of bubbles, bed expansion, pressure signals and time averaged solids or gas volume fractions. The literature on this topic is abundant; from the earlier works of Boemer et al. (1998), and Van Wachem et al. (1998), to the more recent studies comprising image analysis (Busciglio et al., 2009), particle drag optimisation procedures (Mahinpey et al., 2007; Vejahati et al., 2009), and time averaged volume fraction (Deza et al., 2009; Min et al., 2010; Wang et al., 2010). Particularly, the works of Deza et al. (2009) and Min et al. (2010) show a reasonable agreement between two dimensional (2D) and three dimensional (3D) simulations and X ray imaging experiments of cylindrical fluidized beds of internal diameter of 0.095 and $0.152 \mathrm{~m}$, respectively. Wang et al. (2010) compared their $3 \mathrm{D}$ simulation results of time average solids volume fraction in a bubbling bed with experimental data from $\gamma$ ray absorption, capa citance probe and differential pressure measurements, and showed that industrial scale fluidized beds can be reasonable predicted with acceptable computational cost. It is worth noticing that, in con cordance with the two phase theory of gas fluidized beds (Davidson and Harrison, 1963), the velocity and growth of bubbles in a gas fluidized bed primarily depends on the emulsion equivalent density (related to void fraction) and the excess of gas, being less relevant other factors such as the size of the bed and the dense phase motion (Kunii and Levenspiel, 1991). Besides, several studies demonstrate that the simplified two phase theory describes fairly well the behaviour of bubbles in three and two dimensional beds (see for example Darton et al., 1977; Shen et al., 2004).

However, it seems necessary to extend the classical bubble behaviour validation to other macroscopic characteristics such as the mean particle motion. The mean particle motion is a key factor in the motion and mixing of small and large particles (e.g. biomass) in bubbling fluidized beds (Kunii and Levenspiel, 1991). With the development of CCD cameras and Particle Image Velocimetry (PIV) or other velocimetry techniques, there are several studies showing experimental information on particle velocity in very thin, fluidized beds, whose behaviour can be considered two or quasi two dimensional (e.g. Bokkers et al., 2004; Liu et al., 2005; Santana et al., 2005; Almendros Ibáñez et al., 2006; Laverman et al., 2008; Sánchez Delgado et al., 2010). At this regard, reported mean particle velocities are clearly smaller than the characteristic bubble velocity in two dimen sional beds working with Geldart B particles and superficial velocity around 2 times the minimum fluidisation velocity $U_{m f}$. For example, tracking the individual particle trajectories in a quasi two dimensional bed of $0.022 \mathrm{~m}$ thickness, Jung et al. (2005), measured the time averaged vertical velocity of particles far from the lateral walls, obtaining an ascending vertical velocity of value around $0.12 \mathrm{~m} / \mathrm{s}$. Using PIV in a two dimensional bed of $0.015 \mathrm{~m}$ thickness, Laverman et al. (2008), obtained peak velo cities over 0.075 and $0.11 \mathrm{~m} / \mathrm{s}$ for, respectively, the upward and downward mean flow of particles at a vertical distance of $0.15 \mathrm{~m}$ from the distributor. These values are for beds of relatively small lateral width (i.e. $0.15 \mathrm{~m}$ ) at $0.140 .15 \mathrm{~m}$ height over the dis tributor, being the particle velocities smaller if the bed width is increased (Laverman et al., 2008). It seems, however, that the vertical velocity of particles in three dimensional beds (Lin et al., 1985) can be larger than in two dimensional beds, especially for superficial velocities of more than 3 times $U_{m f}$.

Inspection of open literature reveals that, only recently, a reduced number of works make a comparison between the mean particle motion of experiments and two fluid model simulations. Jung and Gidaspow (2006), and also Dan et al. (2010), presented simulation results of a gas fluidized bed with $0.022 \mathrm{~m}$ thickness and $0.15 \mathrm{~m}$ width. Among other results, they compared the simulated time averaged particle velocity with previous experi ments of a similar bed (Jung et al., 2005). The comparison was performed at a height of $0.14 \mathrm{~m}$ from the distributor in the central part of the bed plane (i.e. far from the lateral walls), and the level of agreement shown was excellent. Lindborg et al. (2007) made a qualitative comparison of the velocity vector fields of two dimensional simulations and three dimensional measurements taken from Lin et al. (1985). This was equally done by Hosseini et al. (2010), incorporating also in the comparison the velocity vectors from Laverman et al. (2008). These results evidence a clear resemblance in the particle recirculation patterns obtained in simulation and experiments, but it appears that the simula tions over predict the modulus of the particle velocity vectors for small superficial velocities $\left(U / U_{m f}=1.65\right.$ in Lindborg et al., 2007), and that the prediction of the vertical position of the recirculation centre is not completely satisfactory (Hosseini et al., 2010). 
Ahuja and Patwardhan (2008), for $U / U_{m f}=5.6$, as well as Wang and Liu (2010), this last study using FCC particles, compared their two dimensional simulation results with three dimensional experiments and found acceptable agreement in the radial pro files of velocity. Very recently, Li et al. (2010) compared the experimental results from the work done by Laverman et al. (2008) in a pseudo 2D fluidized bed with 2D and 3D simulations using the drag model of Gidaspow. Their results, accounting for a bed column of $0.015 \mathrm{~m}$ thickness and $0.3 \mathrm{~m}$ width, indicate that there is a great discrepancy with the experimental particle velocities when the system is modelled as a pure $2 \mathrm{D}$ plane due to the lack of interaction with the frontal and rear walls. Note that, for the quantitative comparison, these works employed experimental results from $3 \mathrm{D}$ beds or quasi $2 \mathrm{D}$ beds of thickness greater than 25 times the mean particle diameter and relatively small widths. The reported studies comparing the simulated and the experimental particle velocities devote most of their analyses to the vertical velocity component along a horizontal line in the bed.

Despite the available experimental techniques, none of the above described studies on simulation verification and validation have performed a systematic quantitative comparison of two fluid models and PIV results in the whole plane of a two dimensional bed combining reduced thickness (less than $0.01 \mathrm{~m}$ ), relatively large width, and a small superficial velocity $\left(U / U_{m f}<2\right)$. Such comparison would be interesting since it could be used, not only as a practical validation tool for the two fluid model employed, but also as a complementary source of information in the interpretation of PIV data.

The present work performs a comparison between Eulerian Eulerian two fluid simulation and experimental results of a two dimensional gas solid fluidized bed working in bubbling regime. In particular, thanks to the use of non intrusive techniques based on digital image analysis (DIA), the time averaged distribution, size and velocity of bubbles taken from experiments in a quasi two dimensional bed of reduced thickness are compared with the simulation results of the same bed computed with several implementations of two fluid models. Furthermore, this compar ison is extended to the particle phase velocity outside bubbles, which, in the case of the experiments, is obtained after applying particle image velocimetry (PIV) techniques. The simulation comprises two different closures: Gidaspow (Gidaspow, 1994) and Syamlal \& O'Brien (Syamlal and O'Brien, 1987) drag models. All the closure models are used in standard form to reveal their original behaviour, and no adjustment of coefficient is then performed. The final aim of this multiple comparison is to show whether the level of similarity between simulation and experi ments encountered in bubble kinetics is also present, or not, in particle phase velocity. Unexpectedly, the results obtained in the present work seem to indicate that, despite the reasonable agreement reached in bubble size and velocity, any of the Eulerian Eulerian two fluid models tested overpredicts the mean particle velocity by nearly an order of magnitude compared to the experimental PIV results.

\section{Experimental facility and data acquisition}

The experimental facility used for the comparison of the computational data is a two dimensional cold fluidized bed of dimensions $0.5 \mathrm{~m} \times 2 \mathrm{~m} \times 0.005 \mathrm{~m}$ (width $W$, height $h$, and thick ness $Z$ ) (Sánchez Delgado et al., 2010). Fig. 1 shows a schematics of the facility. The fluidized bed was filled with ballotini glass particles of Geldart B behaviour (Geldart, 1973), $2500 \mathrm{~kg} / \mathrm{m}^{3}$ density, and $600800 \mu \mathrm{m}$ diameter. The fixed bed height was $h_{1}=0.3 \mathrm{~m}$, and the superficial velocity at minimum fluidisation conditions was $U_{m f}=0.35 \mathrm{~m} / \mathrm{s}$ ). A very small fraction of the

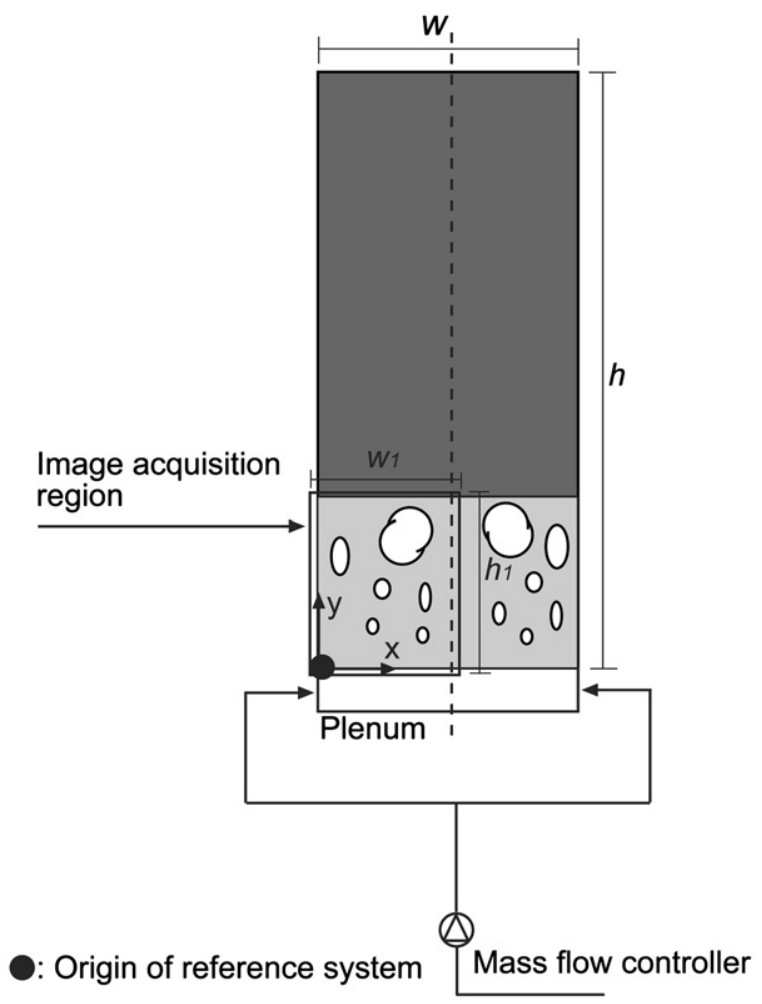

Fig. 1. Schematic of the 2D gas-fluidized bed tested.

particles (less than $1 \%$ ) were black instead of transparent in order to enhance the tracking of the solids phase by improving the peak detection in the PIV correlation matrix. That means that in the tested bed there are less than 9 particles in the bed thickness direction, which ensures the 2D dimensionality of the bed (Nedderman and Laohakul, 1980). Therefore, in principle, 3D effects such as velocity components and gradients along the thickness direction are not going to appear and are not needed to be included in the simulation. The air distributor consists of a perforated plate with 50 holes of $0.001 \mathrm{~m}$ diameter spaced $0.01 \mathrm{~m}$ apart. The front wall was made of glass and the rear wall was made in aluminium, covered by a black card to increase the contrast in the images. The superficial gas velocity $\left(U_{0}=0.62 \mathrm{~m} / \mathrm{s}\right)$ was fixed to 1.75 times $U_{m f}$.

Two $650 \mathrm{~W}$ spotlights were used to get a uniform illumination of the bed. A high speed camera, Redlake Motion pro X3, took images of the front view of the fluidized bed at 125 frames per second. In order to increase the spatial resolution of the images for bubble probability and, especially, dense phase velocity, only half of the bed was recorded $\left(\left[w_{1} \times h_{1}\right][992 \times 1024\right.$ pixels $\left.]\right)$. Fig. 2a shows an example of the image of particles taken from one half on the bed in which the dark regions are the ascending bubbles. From the statistical point of view, the fluidized bed was symme trical in behaviour to the recorded area. On the other hand, to calculate the geometrical properties of bubbles such as equivalent diameter and velocity, it is not needed a great resolution, so bubbles data can be obtained from images taken from the entire bed.

The images from this experimental set up were processed by means of DIA techniques, performed in Matlab R2008a, to obtain the instantaneous bubble size as well as the bubble velocity (i.e. velocity vectors) within the two dimensional domain. Parti cularly, size and centroid position were extracted from the images after performing a threshold that separated gas from dense phase 
a

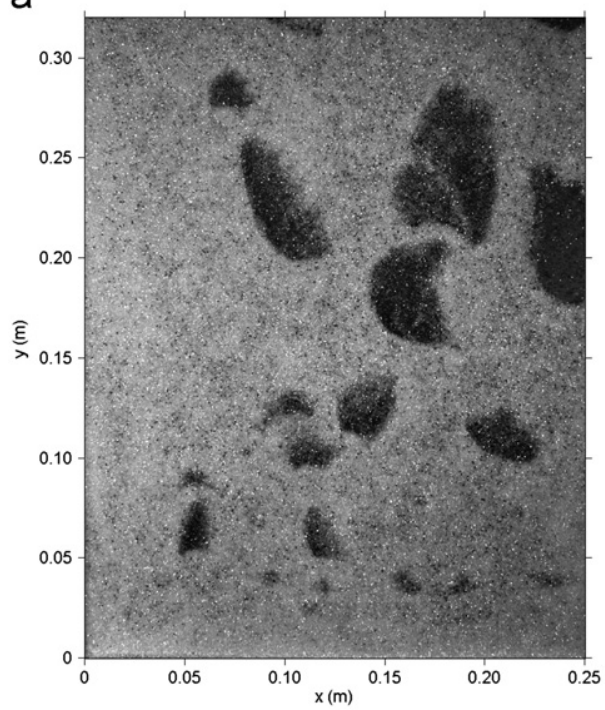

b

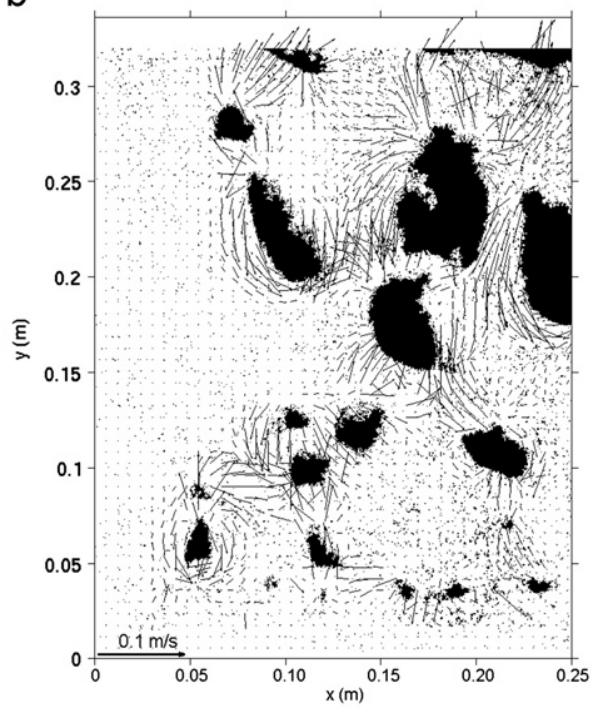

Fig. 2. Processing of experimental results: (a) snapshot of the bubbling fluidized bed and (b) bubble mask and PIV velocity field of the dense phase.

(see Otsu, 1979). Therefore, each pixel of each image was classified as bubble $(C=0)$ or dense phase $(C=1)$. This is represented in Fig. $2 \mathrm{~b}$ as black and white areas, respectively. The experimental uncertainty associated with the bubble contour and centroid position is $2.5 \mathrm{e} 4 \mathrm{~m}$, which is the spatial resolution in the particle image calculated as half the size of a pixel. This uncertainty constitutes a $0.5 \%$ of the typical bubble size in the bed (i.e. $0.05 \mathrm{~m}$ ).

The processed images were summed and rescaled with the total number of images processed, producing a time averaged image that represents the proportion of time that a point is occupied by solid, $\bar{C}(x, y)=\sum_{i}^{N}{ }_{1} C_{i}(x, y) / N$, where $N$ is the number of images, therefore, the proportion of time that a point is occupied by bubbles is defined as $\bar{B}=1 \quad \bar{C} . \bar{B}$ and $\bar{C}$ may be, respectively, interpreted as the bubble and dense phase probability.

Regarding the dense phase velocity, it was calculated by means of a particle image velocimetry (PIV) technique applied to the images. The particular implementation of the technique is also explained in Sánchez Delgado et al. (2010), and is based on the correlation of consecutive in time images using the multigrid PIV code MATPIV (Sveen, 1998 2007). Interrogation windows of $16 \times 16$ pixels with 0.5 overlap were typically used in this PIV analysis performed to compute the velocity field of the dense phase. Following the procedure of Laverman et al. (2008), the time averaged velocity was calculated as $V(x, y)=\sum_{i}^{N}{ }_{1} C_{i}(x, y) v_{s, i}(x, y) / \sum_{i}^{N}{ }_{1} C_{i}(x, y)$ to correct the influence of particle raining from the roof of bubbles. Since $C$ is null inside bubbles, it is only necessary to obtain the particle velocities outside them. Note that bubbles are regions relatively free of particles and the PIV results there may not be reliable Therefore, mean velocities are calculated from PIV results outside bubbles after applying a mask that rejects dense phase velocity inside bubbles. This is indicated in Fig. 2b, which contains an example of the instanta neous velocity vector field of the dense phase. For illustrative purposes the number of vectors per unit area has been reduced in this figure. According to the standard theory of PIV measurement (Raffel et al., 2007), the estimation of each velocity vector can be affected by bias and subpixel errors. In a uniformly illuminated bed, bias errors are principally generated by velocity gradients whose scale is smaller than the interrogation window. In the present work the bias error has been reduced thanks to the use of multigrid PIV techniques that employ very small interrogation windows at the final PIV processing stage. The size of these windows is $16 \times 16$ pixels (about $8 \mathrm{~mm}$ size), which is shorter than the principal velocity scales of the problem. Peak locking and background noise are the main source of subpixel error in a two dimensional velocity field. As the concentration of particles in the dense phase is high, the background noise and peak locking are not expected to have a relevant impact in the accuracy of the results (Raffel et al., 2007). All these effects, together with the unsteady character of the bed, contribute to the uncertainty of the time averaged results. One way to quantify an upper bound for the overall uncertainty of the time averaged results is to evaluate the standard deviation of the average from a series of independing data blocks (Laverman et al., 2008). In the present study, data blocks containing each one over 1000 image pairs were used and compared with a long term average. Following this procedure, the upper bounds of the uncertainty for the time average vertical velocity, horizontal velocity and dense phase probability are $4.84 \%$, $11 \%$ and $1 \%$, respectively. The larger uncertainties are typically obtained near the bed surface. These uncertainties resulted similar to the standard error of the mean, which is obtained as the standard deviation of the whole data set, divided by the square root of the total number of images.

\section{Computational model}

The numerical simulation, performed by solving the conserva tion equations of mass and momentum and granular temperature using FLUENT 6.3.26 CFD software. A multifluid Eulerian model was applied, where gas and solids (i.e. particles) are treated as two interpenetrating phases. The kinetic theory of granular flow, which deals with the conservation of the stochastic fluctuation of solids kinetic energy, was used for the closure of the solids stress terms. Two drag models were employed to solve the interaction between both phases: Gidaspow drag function (Gidaspow, 1994) and Syamlal O'Brien drag function (Syamlal and O'Brien, 1987, 1989). Gidaspow drag model was selected due to the robustness in convergence that it showed during the simulation campaign of the present study. In the Gidaspow model, the kinetic viscosity of solids, $\mu_{s, k i n}$, and the diffusion coefficient of granular temperature, $k_{\Theta}$, from Gidaspow (1994), are also used in the simulation. The simulation with Syamlal \& O'Brien drag function, with $\mu_{s, k i n}$ and $k_{\Theta}$ also from Syamlal et al. (1993). Previous studies (Taghipour et al., 2005) have shown little differences between Gidaspow and Syamlal O'Brien model, nevertheless, simulations with this two 
drag models have been carried out in order to compare them. The governing equations can be summarised as follows.

Mass conservation of gas $(\mathrm{g})$ and solid $(\mathrm{s})$ phases:

$\frac{\partial}{\partial t}\left(\alpha_{g} \rho_{g}\right)+\nabla \cdot\left(\alpha_{g} \rho_{g} \overrightarrow{v_{g}}\right)=0$

$\frac{\partial}{\partial t}\left(\alpha_{s} \rho_{s}\right)+\nabla \cdot\left(\alpha_{s} \rho_{s} \overrightarrow{v_{s}}\right)=0$

Momentum conservation equations of gas and solid phases:

$\frac{\partial}{\partial t}\left(\alpha_{g} \rho_{g} \overrightarrow{v_{g}}\right)+\nabla \cdot\left(\alpha_{g} \rho_{g}{\overrightarrow{v_{g}}}^{2}\right)=\alpha_{g} \nabla p+\nabla \cdot \overline{\overline{\tau_{g}}}+\alpha_{g} \rho_{g} \vec{g} \quad K_{g s}\left(\overrightarrow{v_{g}} \quad \overrightarrow{v_{s}}\right)$

$\frac{\partial}{\partial t}\left(\alpha_{s} \rho_{s} \overrightarrow{v_{s}}\right)+\nabla \cdot\left(\alpha_{s} \rho_{s}{\overrightarrow{v_{s}}}^{2}\right)=\alpha_{s} \nabla p \quad \nabla p_{s}+\nabla \cdot \overline{\overline{\tau_{s}}}+\alpha_{s} \rho_{s} \vec{g}+K_{g s}\left(\overrightarrow{v_{g}} \quad \overrightarrow{v_{s}}\right)$

Granular temperature, $\Theta$, conservation like equation:

$$
\begin{aligned}
& \frac{3}{2}\left[\frac{\partial}{\partial t}\left(\rho_{s} \alpha_{s} \Theta\right)+\nabla \cdot\left(\rho_{s} \alpha_{s} \overrightarrow{v_{s}} \Theta\right)\right]=\left(p_{s} \overline{\bar{I}}+\overline{\overline{\tau_{s}}}\right): \nabla \overrightarrow{v_{s}} \\
& \quad+\nabla \cdot\left(k_{\Theta} \nabla \Theta\right) \\
& \gamma_{\Theta} 3 K_{g s} \Theta
\end{aligned}
$$

where $\left(p_{s} \overline{\bar{I}}+\overline{\overline{\tau_{s}}}\right): \nabla \overrightarrow{v_{s}}$ is the generation of $\Theta$ by the solids stress tensor $k_{\Theta} \cdot \nabla \Theta$ is the diffusion of $\Theta$ energy, $\gamma_{\Theta}$ is the collisional dissipation of $\Theta$ and $3 K_{g s} \Theta$ is the transfer of kinetic energy. In mass and momentum conservation, the code solves the equation for one phase, and calculates the other assuming that $\alpha_{g}=1 \alpha_{s}$. The closure expressions for $K_{g s}, \overline{\overline{\tau_{s}}}, p_{s}, k_{\Theta}$ and $\gamma_{\Theta}$ can be found in Appendix A.

A second order scheme was used to discretized the convective derivatives: Second order implicit for time advance formulation, Phase Coupled SIMPLE for pressure velocity coupling, Second Order Upwind for Momentum and Granular Temperature, QUICK for volume fraction. The 2D computational domain was discre tized using 20301 nodes in a structured but inhomogeneous mesh of rectangular cells. The mesh size has been chosen on the basis of a sensitivity analysis on the mesh density shown in Section 4.1. A time step of $5 \mathrm{e} 4 \mathrm{~s}$, with 75 iterations per time step, was chosen in order to ensure the convergence of the problem, with a maximum residual criterion (as defined in Fluent 6.3 (Fluent, 2006)) of 5e 4 for all equations. 75 iterations per time steps resulted in a conservative figure that ensured, by far, that in each time step the obtained solution was converged, below the maximum residual limit, and did not change if more time steps were added. The Syamlal \& O'Brien drag function was solved with the same time step, but 100 iterations per time step were needed in order to ensure its convergence. The restitution coefficient implemented was 0.9 , which is an acceptable value reported in the literature for glass particles (Syamlal and O'Brien, 1987). Note also that this value includes the effects of energy dissipation due to the particles inelastic deformation and frictional losses (Goldschmidt et al., 2001).

A laminar regime for the gas phase and the walls were modelled employing the standard no slip boundary condition for both phases. This approximate condition ensures that particle velocity profiles are in good agreement with the experimental results (see Section 4). A simple pressure boundary condition was imposed at the top of the freeboard (outlet vent with null lost coefficient). The inlet gas velocity was modelled with a uniform profile, which can be considered similar to that appearing in porous plate distributors and a first approximation for perforated plate distributors. The initial solids volume fraction chosen for the simulation starting was $\alpha_{s}=0.6$, and the selected maximum packing limit is $\alpha_{s, \max }=0.63$. The particle diameter selected corresponds to the averaged diameter of particles used in the experimental set up described in Section 2. More information
Table 1

Main simulation parameters.

\begin{tabular}{ll}
\hline Parameter & Value \\
\hline Particle density, $\rho_{s}$ & $2500 \mathrm{~kg} / \mathrm{m}^{3}$ \\
Gas density, $\rho_{g}$ & $1.225 \mathrm{~kg} / \mathrm{m}^{3}$ \\
Particle diameter, $d_{p}$ & $700 \mu \mathrm{m}$ \\
Restitution coefficient, $e_{s s}$ & 0.90 \\
Initial solids volume fraction, $\alpha_{m f}$ & 0.60 \\
Bed width, $W$ & $0.5 \mathrm{~m}$ \\
Bed height, $h$ & $2 \mathrm{~m}$ \\
Static bed height, $h_{1}$ & $0.3 \mathrm{~m}$ \\
Drag function & Gidaspow, Syamlal-O'Brien \\
Wall boundary condition & No-slip, partial-slip, free-slip \\
Inlet boundary condition & Velocity inlet, 0.62 m/s \\
Outlet boundary condition & Outlet vent \\
Regime & Laminar \\
Time step & $5 \mathrm{e}-4 \mathrm{~s}$ \\
\hline
\end{tabular}

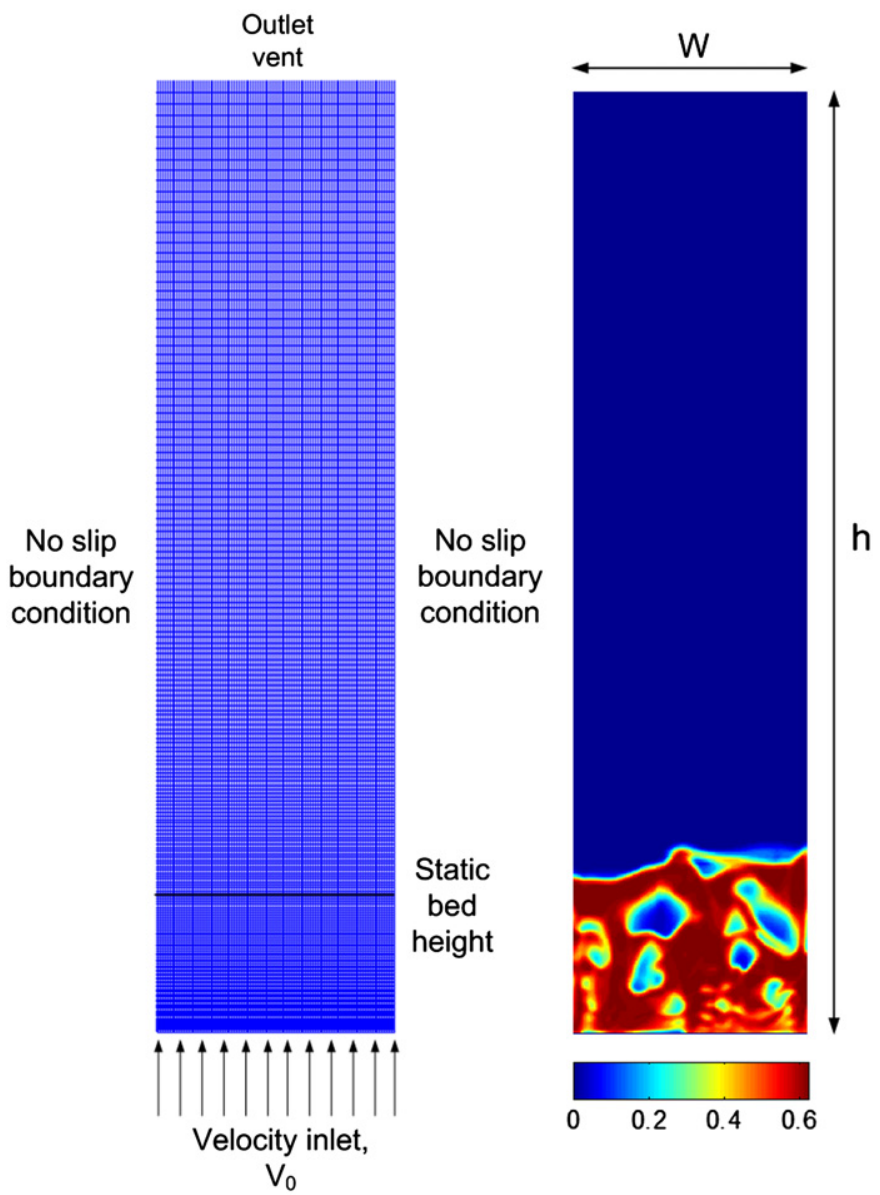

Fig. 3. Computational domain with the boundary conditions used in the simulations (left) and a snapshot of solids volume fraction in the bed after $20 \mathrm{~s}$ of simulation (right)

on the geometrical and operative conditions of the simulated fluidized bed can be encountered in Table 1 .

Fig. 3 illustrates the computational domain employed in the present study, which involves only the two dimensional fluidized bed, that is to say, without considering the plenum and the distributor, and its dimensions correspond to the experimental facility $(0.5 \mathrm{~m} \times 2 \mathrm{~m})$. As Fig. 3 shows, the rectangular grid concentrates toward the inlet in order to improve the spatial resolution in the bubble formation region. Note that, due to the stochastic nature of the instantaneous bubble distribution in the fluidized bed, the computational domain covers all the bed 
volume and not only the half plane used for the experimental data acquisition. In this figure the computational mesh is presented with its boundary condition together with a snapshot of the solids volume fraction obtained after simulating a time period of $20 \mathrm{~s}$.

\section{Results comparison}

The post processed data from the simulation have been compared with the experimental data. The time used for aver aging the experimental data is $26 \mathrm{~s}$, excluding the transient fluidisation start, with a frame rate of $125 \mathrm{~Hz}$ which gives a total number of 3271 images. For the calculation of the time averaged quantities $35 \mathrm{~s}$ were simulated. The first $5 \mathrm{~s}$ of the simulation startup were removed in order to eliminate the initial transient behaviour of the bed. By this way, the actual time period employed for the evaluation of the time average quantities in the simulation was $30 \mathrm{~s}$, which is comparable to the time period used in the experiments. In the simulation, data was exported every 5e 3 real time seconds, which gives 6000 frames to postprocess. The difference between the number of frames used in the simulation and the experiments should not affect the results because the bed is under statistically steady conditions. The main results of this comparison are described in the following subsections.

It has to be said, that in order to be consistent with the technique employed in the experiments, where raining particles inside bubbles are not taken into account (as done in Laverman et al., 2008), the same technique has been developed with the simulation results. On each frame on either simulation or experi ment, the velocity obtained is imposed to be null inside bubbles.

\subsection{Sensitivity of results on mesh density}

The influence of the number of nodes on the results has been studied for the reference case described in Table 1 . This sensitivity of the results on the mesh density can be considered as indicative of the level of discretization error in the simulation. Fig. 4 contains some examples of time averaged results for the refer ence case (Table 1) using a refined mesh of 80601 nodes and a coarse mesh of 10431 nodes, compared with the simulation results for the mesh of the previous sections (20301). Each result has been normalised with the simulation output of the case using a mesh of 20301 nodes. In the figure, the VOF mean value variable corresponds to the time averaged of the mean dense phase volume fraction over the whole bed plane. Also in Fig. 4 the

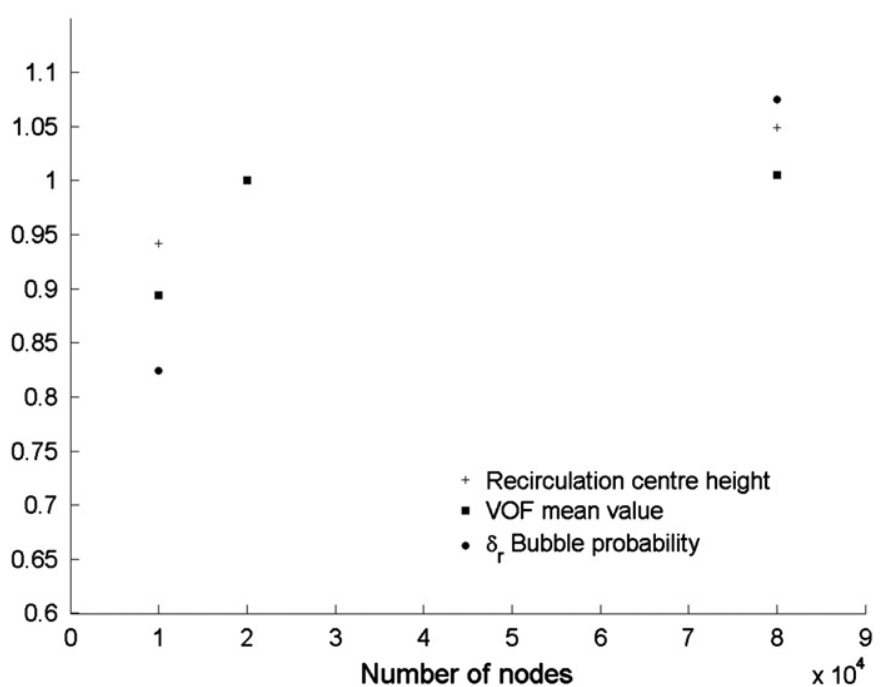

Fig. 4. Sensitivity of time-averaged results on the mesh density.
Table 2

Sensitivity results on the mesh density.

\begin{tabular}{lllll}
\hline $\begin{array}{l}\text { Mesh (number of } \\
\text { nodes) }\end{array}$ & $\min \Delta x(\mathrm{~m})$ & $\begin{array}{l}\max \Delta y \\
(\mathrm{~m})\end{array}$ & $\begin{array}{l}\text { Average } \\
\text { solids } \\
\text { volume } \\
\text { fraction }\end{array}$ & $\begin{array}{l}\text { Compu- } \\
\text { tational } \\
\text { cost }(\mathrm{h})\end{array}$ \\
\hline $10431(57 \times 183)$ & 0.0089 & 0.0039 & 0.4853 & $\sim 100$ \\
$20301(101 \times 201)$ & 0.005 & 0.0028 & 0.5019 & $\sim 200$ \\
$80601(201 \times 401)$ & 0.0025 & 0.0014 & 0.5095 & $\sim 800$ \\
\hline
\end{tabular}

recirculation centre height refers to the mean height of the two main recirculations of solids in the bed (see Section 4.3), and the $\delta$ bubble probability is the discrepancy in bubble probability over the whole bed (Section 4.2). Although some differences between the case of 20301 nodes and the case of 80601 are encountered in Fig. 4 , these differences constitute less than $10 \%$ of the resultant values. In contrast, a mesh of 10431 nodes leads to larger differences in the parameters shown in Fig. 4. The time of computation and the effort required for the image analysis of the refined mesh was more than 4 times that of the mesh for 20301. Thus, a mesh with 80601 nodes yields little changes in the results compared to a mesh of 20301 nodes but much more computational effort.

In addition, the average solids volume fraction in a middle point of the bed $(x=0.25 \mathrm{~m}, y=0.25 \mathrm{~m})$ (Table 2) has been calculated over $30 \mathrm{~s}$ of simulation for the three tested meshes. Also the computational cost (time to complete each computation) is included for a simulated time of $30 \mathrm{~s}$. Taking into account all the results on the grid sensitivity, it seems that the mesh with 20301 provides simulation results very similar to the refined mesh (80601 nodes) while requiring a quarter of computational effort. Therefore, this justifies the use of the mesh with 20301 nodes as the default option in the present work.

\subsection{Bubble probability and time averaged particle velocity}

Fig. 5a and b compares the bubble probability maps with the experimental measurements and the simulation using the para meters of Table 1 . In the construction of the probability maps, the simulation results were processed in the same way as described for the image of particles in Section 2, that is, assuming a value of 0 at the points of the simulated fluidized bed that are inside a bubble and a value 1 otherwise. Note that this methodology eliminates the continuous transition of particle volume fraction that connects the interior to the exterior of the bubbles in the simulated results. A threshold value of 0.3 in particle volume fraction was used to separate the interior from the exterior of a bubble.

The lowest values in bubble probability, for both numerical and experimental results, appear at the bottom of the bed and near the walls. This indicates, as expected, that bubbles are less frequent near the lateral walls of the bed and smaller in size near the distributor. Paying attention to the bubble channelling, it can be observed that the highest bubble probability is near the free surface and also in a vertically aligned region in the bed created by the tendency of bubbles to ascend through the same path (i.e. bubble channel). The bubble channelling is less intense in the computed than in the experimental bed. This indicates that the simulation model of Table 1 provides a better distribution of bubble than the experiment.

One interesting question is whether the bubble probability maps obtained are equivalent or not to the mean void fraction concentration maps. Or, otherwise, it would be useful to know if dense phase probability maps (i.e. one minus the bubble 
a

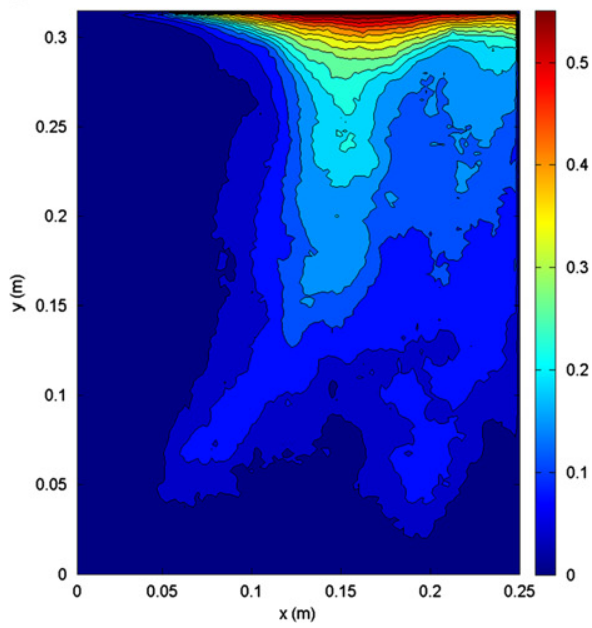

b

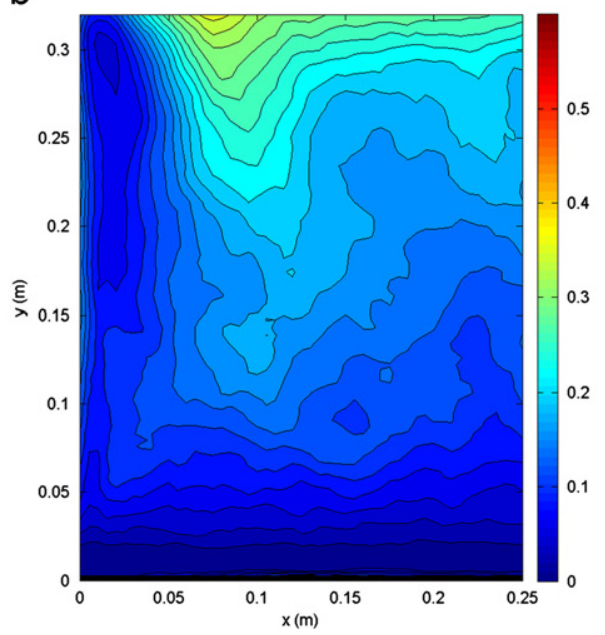

C

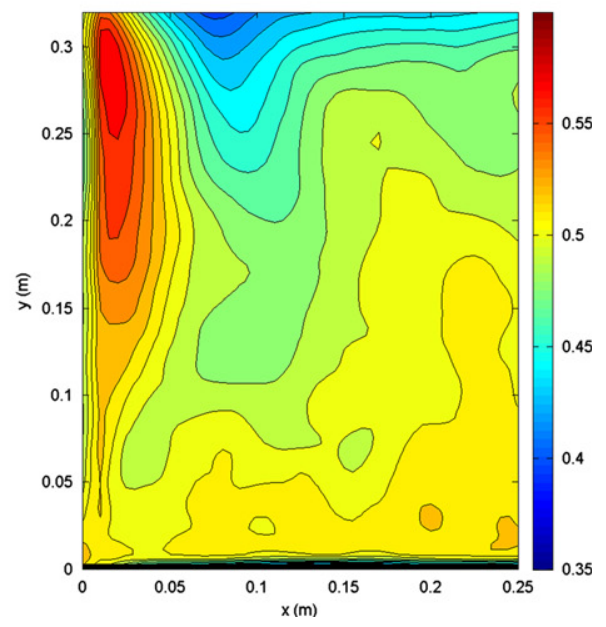

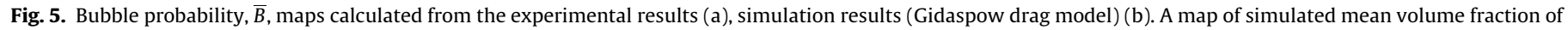
particles, $\overline{\alpha_{s}}$, is also included (c).

probability) may be used to estimate the mean volume fraction of particles in a bubbling fluidized bed. Since the maps of both dense phase probability and mean volume fraction of particle are directly available from the numerical results, the simulation can be used to give an answer to this question. For this purpose, the mean volume fraction of particles, Fig. 5c, has been calculated from the simulation described in Table 1. From the comparison of Fig. 5b and $\mathrm{c}$ it is clear that mean dense phase the two dimen sional patterns of probability distribution and the mean volume fraction of particles are very similar.

A more detailed comparison of bubble and dense phase probability results has been done at different bed heights: near the lower end part of the bed, $y=0.05 \mathrm{~m}$, at the half of the bed, $y=0.15 \mathrm{~m}$ and $0.25 \mathrm{~m}$, and at a region close to the freeboard, $y=0.30 \mathrm{~m}$. In particular, Fig. 6a and c compares the experimental and simulation profiles of bubble probability and mean dense phase vertical velocity, $V_{y}$, for a horizontal cut at $0.25 \mathrm{~m}$ from the distributor. Apart from the drag model of Gidaspow (1994) used as reference case, Table 1 , a complementary simulation is also included in Fig. $6 \mathrm{~b}$ relying on the same operating parameters as the reference case but using the Syamlal \& O'Brien drag model (Syamlal and O'Brien, 1987). In experimental as well as numerical results, the bubble probability increases towards the centre of the bed, i.e. $x=0.25 \mathrm{~m}$ in Fig. $5 \mathrm{a}$, due to the mentioned channelling of bubbles. However, the channelling region is wider in the numer ical results due to a better distribution of bubbles in the computed bed. This effect is present in both models, but seems to be more pronounced in the simulation with the Syamlal \& O'Brien drag model. Interestingly, all the simulations show an increment in bubble probability near the wall that is not observed in experiments. This indicates that the bubbles in the numerical simulation are more prone to attach to the wall than in the experiments.

A comparison of time averaged vertical velocities of solids particles, $V_{y}$, is shown in Fig. 6c, where we can see that although the trends are similar, the magnitude of the numerical velocities are higher, especially when using the drag model from Syamlal \& O'Brien. This fact indicates that the simulated bubbles impel upwardly the dense phase in a more effective way than the actual bubbles in the experimental bed. As a result, sharper gradients of descending $V_{y}$ appear near the wall in the simulation results compared to the PIV measurements. Due to the wide bubble path shown by the simulation, the time averaged downward velocity has a peak value closer to the wall than the experiments. As the experimental time averaged particle velocity is very small com pared to the simulated one, the PIV velocity profiles are nearly flat in Fig. 6c. Particularly, for the downwardly moving mean flow of particles (close to the wall) the minimum velocity is of order $0.02 \mathrm{~m} / \mathrm{s}$, whereas far from the walls the peak of ascending velocity is $0.015 \mathrm{~m} / \mathrm{s}$. Note that these very reduced values of particle velocity are also observed in the work of Laverman et al. (2008). For illustrative purposes, experimental results for $U / U_{m f}=2.5$ are also included in Fig. 6c. These experimental results demonstrate that after increasing the superficial gas velocity from $U=1.75 U_{m f}$ to $2.5 U_{m f}$, the magnitude of time averaged particle velocity remains very small compared to the simulation.

The largest velocity gradients and the velocity peak closest to the wall are found in Fig. 6b for the Syamlal \& O'Brien model. Therefore, in view of Fig. 6a and b, of the two drag models tested in the present two dimensional configuration of fluidized bed, the drag model of Gidaspow seems to yield the closest qualitative results to the experimental profiles of bubble probability and dense phase velocity. However, the discrepancies in magnitude between the time averaged particle velocity of the simulation using Gidaspow drag model and the experimental results for the two dimensional bed of Table 1 are still enormous. When the simulation is performed increasing the restitution coefficient to $e_{s s}=0.99$, the time averaged velocity is reduced up to a $70 \%$ from the $e_{s s}=0.9$, as Fig. $6 \mathrm{~b}$ indicates, but the particle velocity overestimation continues to be very appreciable and the simulated profiles of bubble probability clearly separates from the experiments for $x>0.1 \mathrm{~m}$ (Fig. 6a). The time averaged velocity of particles is also diminished by reducing the initial solids volume fraction of the simulation to an arbitrary small value $\alpha_{s}=0.55$, instead of the more realistic bed parameter $\alpha_{s}=0.6$, and maintaining the rest of operative conditions of Table 1 . As in the case of increasing the coefficient of restitution, the price to be paid with the diminishing of $\alpha_{s}$ is the reduction of the simulated bubble probability near the bed centre $(x=0.25 \mathrm{~m})$. Furthermore, it seems that increasing $e_{s s}$ or $\alpha_{s}$, also eliminates the qualitative agreement between the velocity profiles. This is shown in Fig. 6b, in which the velocity profiles for the simulation using Gidaspow drag model with $e_{s s}=0.99$ or $\alpha_{s}=0.55$ are characterised by a downflow motion of particles at $x=0.25 \mathrm{~m}$ that is more intense than the obtained for original parameters $e_{s s}=0.9$ and $\alpha_{s}=0.6$.

Fig. $6 \mathrm{~b}$ also illustrates that the no slip boundary condition imposed in the simulation for the solids phase is as approximately verified in the experimental results. However, the region in which 

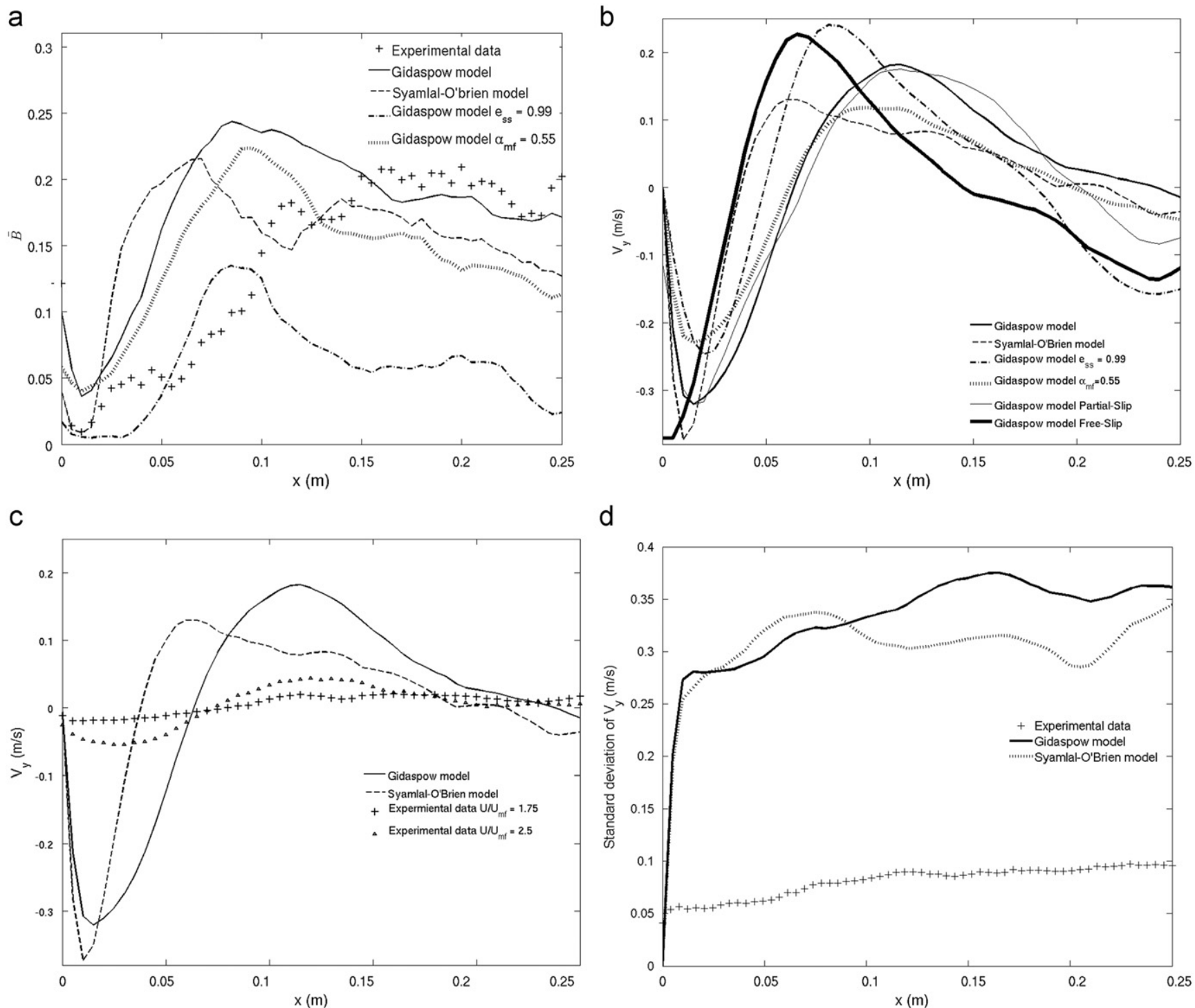

d
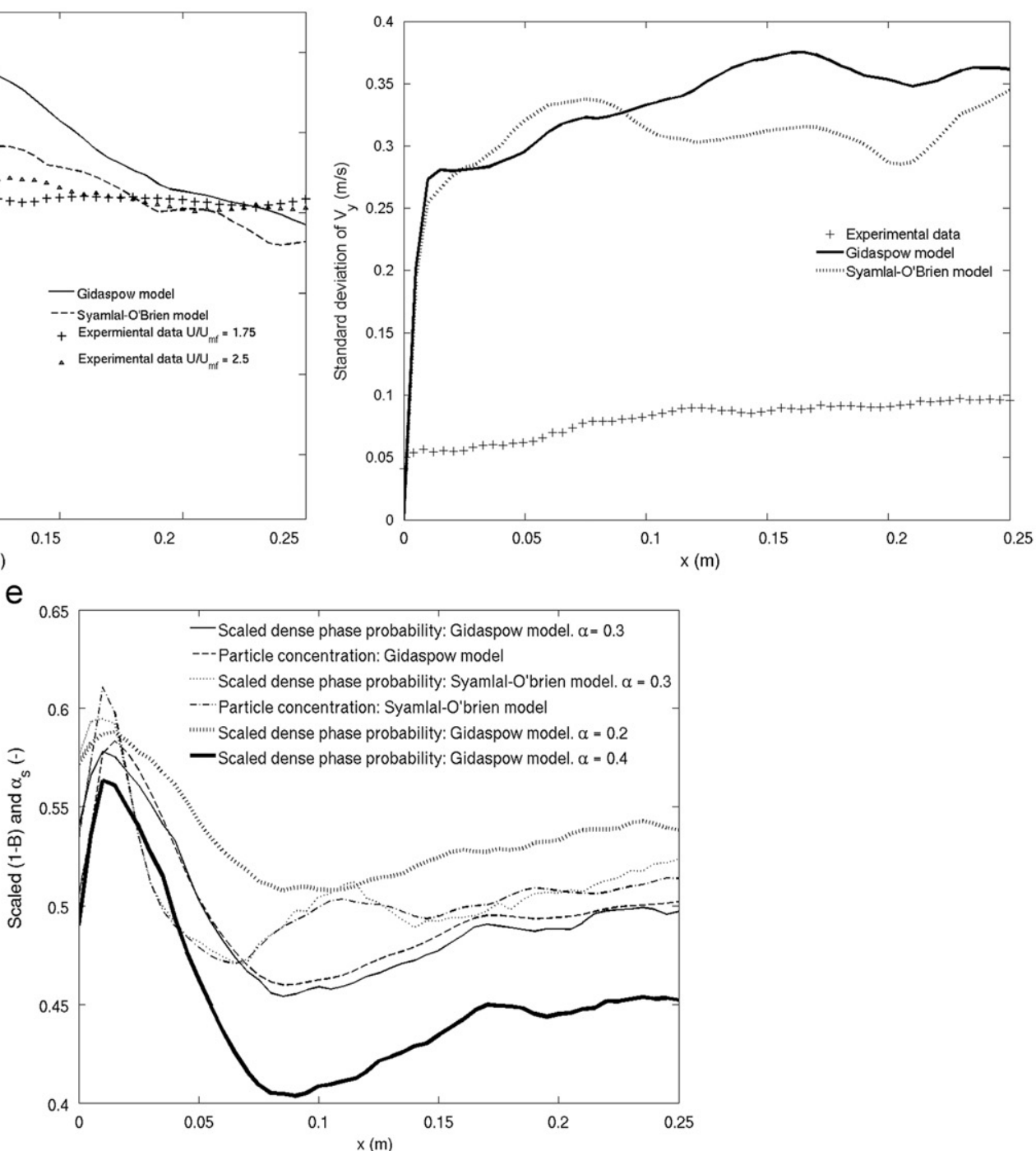

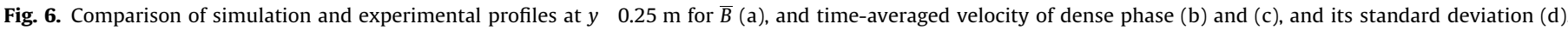
Also, included the comparison of scaled dense phase probability, $\bar{C} \alpha_{m f}$, and mean solids volume fraction, $\overline{\alpha_{s}}$, calculated from the simulation (e).

descending particles are slowed down by the lateral walls is larger in the experiments than in the simulation, suggesting that perhaps other effects such as frontal and posterior walls friction are influencing the measured bed in this region. Profiles for two different lateral boundary conditions have been included, i.e. free slip (specularity coefficient $=0$ ) and partial slip (specularity 
Table 3

Discrepancy of measurements from simulation results using Gidaspow drag function.

\begin{tabular}{|c|c|c|c|c|c|c|c|c|}
\hline \multirow[t]{2}{*}{ Variable } & \multicolumn{2}{|l|}{$y \quad 0.05 \mathrm{~m}$} & \multicolumn{2}{|l|}{$y \quad 0.15 \mathrm{~m}$} & \multicolumn{2}{|l|}{$y \quad 0.25 \mathrm{~m}$} & \multicolumn{2}{|l|}{$y \quad 0.30 \mathrm{~m}$} \\
\hline & $\delta$ & $\delta_{r}(\%)$ & $\delta$ & $\delta_{r}(\%)$ & $\delta$ & $\delta_{r}(\%)$ & $\delta$ & $\delta_{r}(\%)$ \\
\hline $\bar{C}$ & 0.0591 & 6.15 & 0.0399 & 4.52 & 0.0707 & 8.17 & 0.1287 & 15.21 \\
\hline $\bar{B}$ & 0.0591 & 138.18 & 0.0399 & 32.33 & 0.0707 & 46.36 & 0.1287 & 70.91 \\
\hline$V_{y}$ & $0.0119 \mathrm{~m} / \mathrm{s}$ & 136.59 & $0.0554 \mathrm{~m} / \mathrm{s}$ & 657.43 & $0.1166 \mathrm{~m} / \mathrm{s}$ & 944.34 & $0.0993 \mathrm{~m} / \mathrm{s}$ & 748.77 \\
\hline$V_{x}$ & $0.0173 \mathrm{~m} / \mathrm{s}$ & 439.35 & $0.0554 \mathrm{~m} / \mathrm{s}$ & 1088.33 & $0.0374 \mathrm{~m} / \mathrm{s}$ & 1355.25 & $0.0592 \mathrm{~m} / \mathrm{s}$ & 2334.65 \\
\hline
\end{tabular}

Table 4

Discrepancy of measurements from simulation results using Syamlal \& O’Brien drag function.

\begin{tabular}{|c|c|c|c|c|c|c|c|c|}
\hline \multirow[t]{2}{*}{ Variable } & \multicolumn{2}{|l|}{$y \quad 0.05 \mathrm{~m}$} & \multicolumn{2}{|l|}{$y \quad 0.15 \mathrm{~m}$} & \multicolumn{2}{|l|}{$y \quad 0.25 \mathrm{~m}$} & \multicolumn{2}{|l|}{$y \quad 0.30 \mathrm{~m}$} \\
\hline & $\delta$ & $\delta_{r}(\%)$ & $\delta$ & $\delta_{r}(\%)$ & $\delta$ & $\delta_{r}(\%)$ & $\delta$ & $\delta_{r}(\%)$ \\
\hline $\bar{C}$ & 0.0429 & 4.46 & 0.0270 & 3.06 & 0.0721 & 8.31 & 0.1188 & 13.93 \\
\hline $\bar{B}$ & 0.0429 & 106.73 & 0.0270 & 21.85 & 0.0721 & 47.33 & 0.1188 & 69.63 \\
\hline$V_{y}$ & $0.0733 \mathrm{~m} / \mathrm{s}$ & 840.50 & $0.1281 \mathrm{~m} / \mathrm{s}$ & 1520.19 & $0.1015 \mathrm{~m} / \mathrm{s}$ & 821.79 & $0.0980 \mathrm{~m} / \mathrm{s}$ & 739.09 \\
\hline$V_{x}$ & $0.0802 \mathrm{~m} / \mathrm{s}$ & 2039.98 & $0.0159 \mathrm{~m} / \mathrm{s}$ & 312.15 & $0.0805 \mathrm{~m} / \mathrm{s}$ & 2918.38 & $0.0630 \mathrm{~m} / \mathrm{s}$ & 2481.40 \\
\hline
\end{tabular}

coefficient $=0.6$ ), in order to ensure that the overestimation of the solids velocity is not a consequence of the lateral walls boundary condition. As it can be seen, the three conditions give velocities in the same order of magnitude and generate the same bed beha viour. Taking this into account, the effects of the lateral walls boundary condition will not be longer discussed in this paper.

Fig. 6d shows the standard deviation of the solids phase velocity shown in Fig. 6b, comprising the experimental results, as well as the simulation results using Gidaspow and Syamlal O'Brien drag models. The standard deviations have been obtained in each point time averaging the squared difference of the instantaneous vertical velocity and its mean value. The velocity standard deviation follows the same trend than the time aver aged values. Note that the simulation gives values an order of magnitude higher than the experiments. Obviously close to the wall, the standard deviation calculated from the simulation tends to zero as no slip conditions were selected. Nevertheless, this happens for a small region near the wall that do not affects the rest of the bed. In the experimental results this decrement of velocity standard deviation is also appreciated but not with the same intensity.

As mentioned previously, in order to compare dense phase probability with mean particle concentration, it is necessary to rescale one of the variables. In Fig. 6e, the horizontal profile of the dense phase probability has been rescaled, by multiplying it by 0.6 , and depicted together with the mean particle concentration from simulations that use Gidaspow and Syamlal \& O'Brien drag models. Clearly, the dense phase concentration is practically equal to the mean volume fraction of particles. This is probably due to the nearly uniformity of the void fraction in the dense phase outside bubbles. In consequence, Fig. 6e encourages the use of the scaled dense phase probability as a good approximation to the mean volume fraction of particles in bubbling fluidized beds.

Fig. 6e illustrates in addition the scaled dense phase prob ability for different bubble detection thresholds. This representa tion clearly shows that using 0.3 as threshold ensures that the simulated dense phase probability $(1 \bar{B})$ is analogous to the simulated time averaged dense phase. The results indicate that the boundary of the bubble placed with threshold $\alpha_{s}=0.3$ perfectly balances de gradients of solids volume fraction towards the interior of the bubble and towards the exterior.
The root mean squared discrepancy along a horizontal line in the bed, $\delta$, can be used in order to obtain a numerical quantifica tion of the differences between simulation and experiments in two dimensional beds:

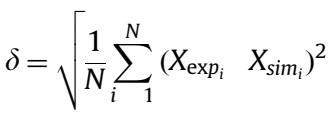

where $X$ corresponds to the variable which is going to be compared and $N$ is the number of data values (i.e. points along the selected horizontal line) included in the calculation of the mean square discrepancy.

Thus, the relative discrepancy of the simulation in reproducing the experimental data is calculated after dividing $\delta$ by the root mean square value of the measured variable:

$\delta_{r}=\frac{\delta}{\sqrt{(1 / N) \sum_{i}^{N}{ }_{1} X_{\exp }^{2}}}$

Tables 3 and 4 summarise the discrepancies encountered between the experimental measurements of the two dimensional bed and the simulation with Gidaspow and Syamlal \& O'Brien drag models. Four different heights have been selected. For the two drag models tested, the tabulated results show that the absolute discrepancy, $\delta$, in dense phase and bubble probability increase with $\mathrm{y}$ for $y>0.05 \mathrm{~m}$. The relative discrepancy in bubble probability is an order of magnitude superior ( $\leq 138 \%$ ) than in dense phase prob ability ( $\leq 15 \%$ ) on account of the reduced root mean square value of bubble probability, especially near the distributor. The relative discrepancy in dense phase velocities is by far larger than in bubble probability, reaching a maximum of $1323 \%$ and $2389 \%$ for vertical and horizontal velocities, respectively. The lowest absolute discre pancies $\delta$ in velocity are typically encountered near the distributor, probability while the largest discrepancies are placed at the centre of the bed height (i.e. $V_{y}$ ) or near the bed surface $\left(V_{x}\right.$ for Gidaspow drag model). As the root mean square value of $V_{y}$ increases rapidly with the distance to the distributor, there is a decrease in the relative value of discrepancy, $\delta_{r}$, for the two drag models. Observe that the relative discrepancy for horizontal velocity $V_{x}$ is usually larger than for $V_{y}$. 
Table 5

Discrepancy between the mean volume fraction of particles and the scaled dense phase probability.

\begin{tabular}{|c|c|c|c|c|c|c|c|c|}
\hline \multirow{2}{*}{$\begin{array}{l}\text { Time-averaged particle concentration vs } \\
\text { scaled dense phase probability }\end{array}$} & \multicolumn{2}{|c|}{$y \quad 0.05 \mathrm{~m}$} & \multicolumn{2}{|c|}{$y \quad 0.15 \mathrm{~m}$} & \multicolumn{2}{|c|}{$y \quad 0.25 \mathrm{~m}$} & \multicolumn{2}{|c|}{$y \quad 0.30 \mathrm{~m}$} \\
\hline & $\delta$ & $\delta_{r}(\%)$ & $\delta$ & $\delta_{r}(\%)$ & $\delta$ & $\delta_{r}(\%)$ & $\delta$ & $\delta_{r}(\%)$ \\
\hline Gidaspow drag function & 0.0372 & 10.38 & 0.005 & 1.40 & 0.0081 & 2.30 & 0.0110 & 3.35 \\
\hline Syamlal-O'Brien drag function & 0.0320 & 8.57 & 0.0099 & 2.65 & 0.0082 & 2.29 & 0.0097 & 2.92 \\
\hline
\end{tabular}

a

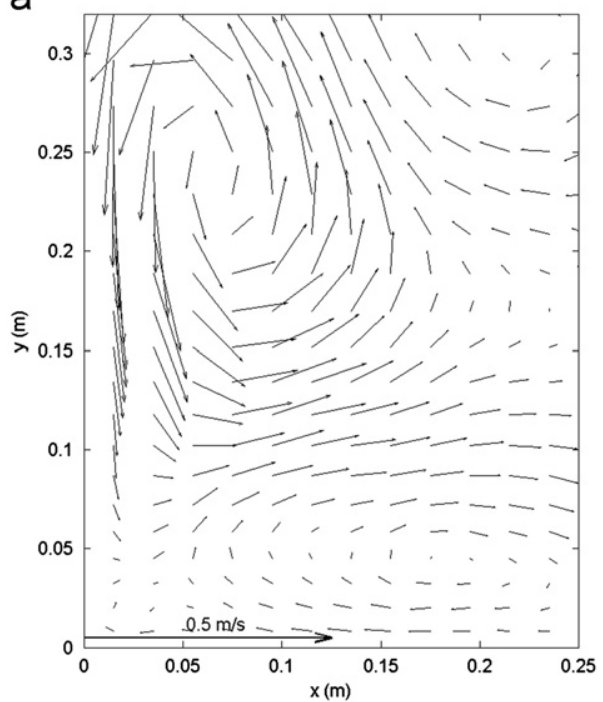

b

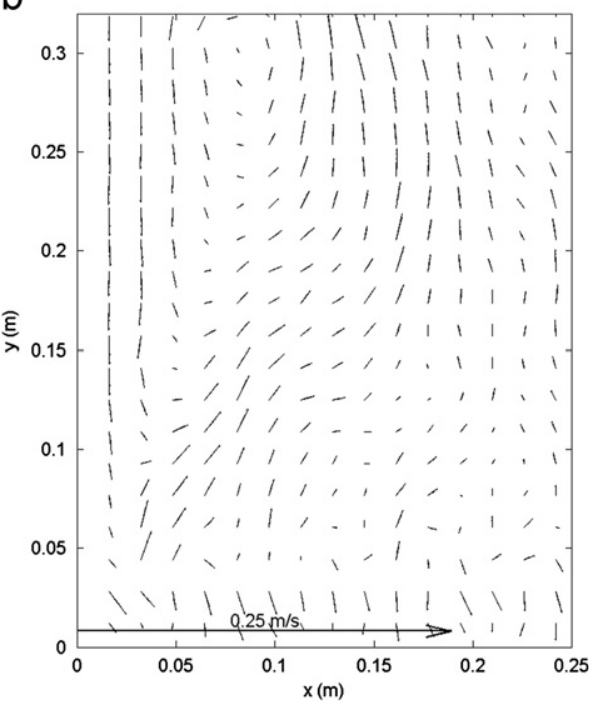

Fig. 7. Time-averaged velocity vectors of dense phase calculated from simulation (a) and experiments (b).

As Tables 3 and 4 shows, Syamlal \& O'Brien drag model produces a slightly smaller discrepancy of the simulated bubble or dense phase probability compared to Gidaspow model. However, regarding the smaller time averaged dense phase velocity, it is the Gidaspow model which yields the discrepancies, especially near the distributor. For brevity reasons, only the simulations for this drag model will be shown in the remaining sections.

Table 5 contains the discrepancies between the mean volume fraction of particles and the scaled dense phase probability, where both magnitudes obtained from simulations. The tabulated results corroborate that the mean volume fraction of particles can be estimated with the scaled dense phase probability, being the estimation discrepancy 3\% near the surface of the bed and around $10 \%$ close to the distributor (i.e. $y=0.05 \mathrm{~m}$ ).

\subsection{Recirculation regions and flow of solids in the time averaged BEd}

The causes of the discrepancies between simulations and experiments encountered in the previous section can be surveyed by comparing the recirculation of particles within the bed. Fig. 7 shows the time averaged velocity field for the simulation in Table 1 and for the PIV results from experiments. To enhance visualisation not all the vectors has been displayed in Fig. 7. It can be observed that the velocities in the upflow and downflow of particles are remarkable larger in the simulation, Fig. 7a, than in the actual fluidized bed, Fig. 7b. Another clear difference is the location of the mean recirculation centres. To visualise more easily the position of the recirculation centres, Fig. 8 presents the streamlines connecting the tangent direction of each time averaged vector field presented in Fig. 7. Although not very rigorously, these streamlines may be interpreted as indicative of the time averaged pathlines of the bed particles. Appendix B details the method used for the computation of the streamlines. It should be noted that the streamlines have been obtained assum ing the dense phase as an incompressible and two dimensional fluid. The incompressibility of the dense phase outside bubbles is a well grounded assumption (Davidson and Harrison, 1963). Of course the bubbles have a perturbation effect on the incompres sibility of the dense phase that is transmitted to the time averaged velocity field. Time averaging the Eq. (1), the incompressibility condition (i.e. $\nabla \cdot \bar{v}_{s}=0$ ) surfaces up since, for the fluidisation regime studied here, the gradients of the time averaged volume fraction of particles are small (except near the bed surface, see Fig. 5) and the coupled oscillation of particle velocity and volume fraction is confined only to the bubble perimeter and bed surface.

Recalling that half of the bed has been studied, Fig. 8 indicates the existence of two main recirculation regions in the bed (i.e. one per half area studied) whose centre position is placed in the simulation nearer to the wall and to the distributor than in the results from the experiments. The location of the recirculation centre is shown in Table 6. This concentrates the experimental streamlines towards the centre line of symmetry $(y=0.25 \mathrm{~m}$ in Fig. $8 \mathrm{~b}$ ) in contrast to the ascending streamlines from the simulation, which are closer to the wall (Fig. 8a). Therefore, in order to preserve the descending flow of particles (streamlines near the wall at $y=0 \mathrm{~m}$ ), the simulation results are characterised by a larger downward velocity than the corresponding experi mental data. According to Table 6 , the descending flow of particles has a peak velocity whose modulus, $\max \left|V_{x}\right|$ and $\max \left|V_{y}\right|$, are an order of magnitude larger in the simulation than in the experiments. Not only is the displacement of the recircula tion region the cause of the large values of $\delta$ in Tables 3 and 4 Calculating the time averaged particle mass flow as the integral along a horizontal line connecting the two main recirculation 
a

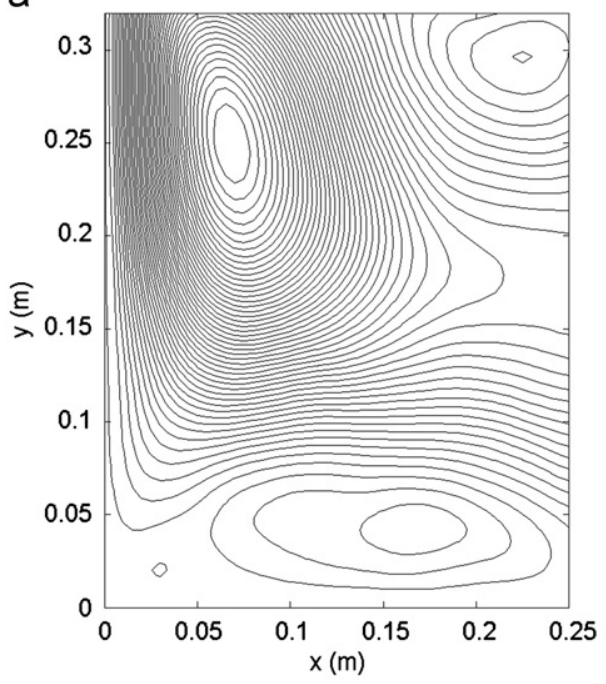

b

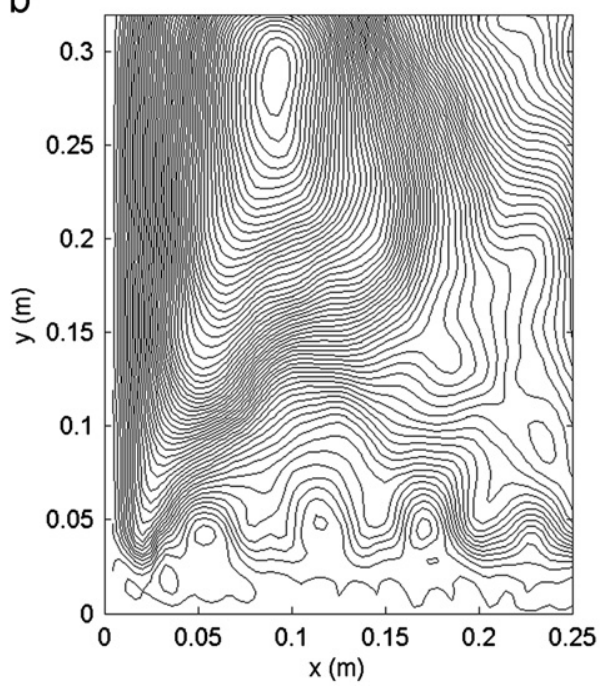

Fig. 8. Streamlines of the time-averaged velocity of dense phase calculated from simulation (a) and experiments (b).

Table 6

Comparison of time-averaged results for the dense phase flow in the bed.

\begin{tabular}{lll}
\hline Variable & Numerical & Experimental \\
\hline$x_{R C}(\mathrm{~m})$ & 0.0700 & 0.0927 \\
$y_{R C}(\mathrm{~m})$ & 0.25063 & 0.2863 \\
$\max \left|V_{x}\right|(\mathrm{m} / \mathrm{s})$ & 0.2190 & 0.0212 \\
$\max \left|V_{y}\right|(\mathrm{m} / \mathrm{s})$ & 0.3243 & 0.0348 \\
$m_{p}(\mathrm{~kg} / \mathrm{s})$ & 0.1956 & 0.0243 \\
\hline
\end{tabular}

centres of a bed,

$\dot{m}_{p}=\int v_{s, y} \alpha_{s} \rho_{p} Z d x$

the results in Table 6 evidence that the transport of particles is 10 times larger in the simulation compared to the experiment. As the superficial gas velocity is equal in both cases, one plausible explanation is that the ultimate origin of the discrepancies between simulations and experiments is due to an over predicted net drag force on the ascending dense phase in the two fluid models employed in the present study.

Fig. 8a and b also sheds light into the high discrepancies in time averaged velocity of particles encountered near the distri butor. In the numerical results of Fig. 8a it appears another minor recirculation zone close to the distributor. It seems that in the experiments there is also small intensity recirculations located nearby the distributor but smaller than in the simulation. However, the nature of the minor recirculations near the distributor in the experiments seems to be probably produced by the three dimensionality of particle flow near the distributor. The three dimensional character of the particle flow near the distributor is caused by the bubbles, whose size in that region is smaller or comparable to the thickness of the bed. Of course, the two dimensional simulations cannot reproduce these small recircula tions. Without a doubt, this contributes to the large discrepancies for $V_{x}$ and $V_{y}$ observed in Tables 3 and 4 at $y=0.05 \mathrm{~m}$.

\subsection{Bubble diameter and velocity}

After processing the results from the two phase code, the bubble data can be compared with the experimental data and with theoretical models. The procedure to capture the simulated bubbles is based on a threshold value. Contours of solids volume fraction equal 0.3 are obtained on each frame, and bubble centroid and bubble equivalent diameter are obtained at this point using the contour and avoiding bubbles with a diameter lower than $0.01 \mathrm{~m}$. Bubble velocity is obtained associating bubbles on time step $t$ with the time step $t+1$, taking into account the size of the bubble and its height. This can be easily done because the time step used to analyse the data is small enough to track any bubble properly. The procedure followed by the calculation of bubble diameter and velocity using the simula tion results is exactly the same than using the experimental results once the bubble contour is defined.

The theoretical model used in the present study is the adaptation of the Darton's model (Darton et al., 1977) to two dimensional beds (Shen et al., 2004), in which the diameter of a bubble subjected to coalescence is modelled as

$D_{b}=\left(\frac{5.4543}{\lambda}\right)^{2 / 3}\left[\left(\begin{array}{ll}U & U_{m f}\end{array}\right)\left(y+\frac{\lambda}{2.1419} \frac{A_{0}}{b}\right)\right]^{2 / 3} g^{-(1 / 3)}$

$U_{b}=\varphi \sqrt{ } g D_{b}$

where $\varphi$ is a parameter that takes several values depending on the literature. Davidson and Harrison (1971) gave a value for $\varphi$ equal to 0.71 for three dimensional beds. Later Shen et al. (2004) proposed a value between 0.8 and 1 applicable to two dimensional beds. Also in Eq. (9), $A_{0}$ is the total area of the distributor divided by the number of orifices, $\mathrm{b}$ is the thickness of the bed, $U$ is the superficial velocity of the gas, $U_{m f}$ is minimum fluidisation velocity, $y$ is the vertical distance from the distributor to the bubble centroid, and $\lambda$ is a constant obtained from experiments which for the studied fluidized bed takes a value around 9.86 (Almendros Ibáñez et al., 2006).

Fig. 9 shows the relation between the mean (time average) and standard deviation of the equivalent bubble diameter (Caicedo et al., 2003) versus the vertical distance from the distributor to the bubble centroid, $y$, and Fig. 10 depicts the dependence of the mean and standard deviation of bubble velocity on the equivalent bubble diameter. The simulation and the experimental data compared in these figures are represented by average values (Figs. 9a and 10a) and their standard deviation (Figs. 9b and 10b). In particular, for the construction the average data in Figs. 9a and 10a, the arithmetical mean is calculated with the bubbles whose centroid is placed within a thin interval surrounding each value selected in the horizontal axis. 
a

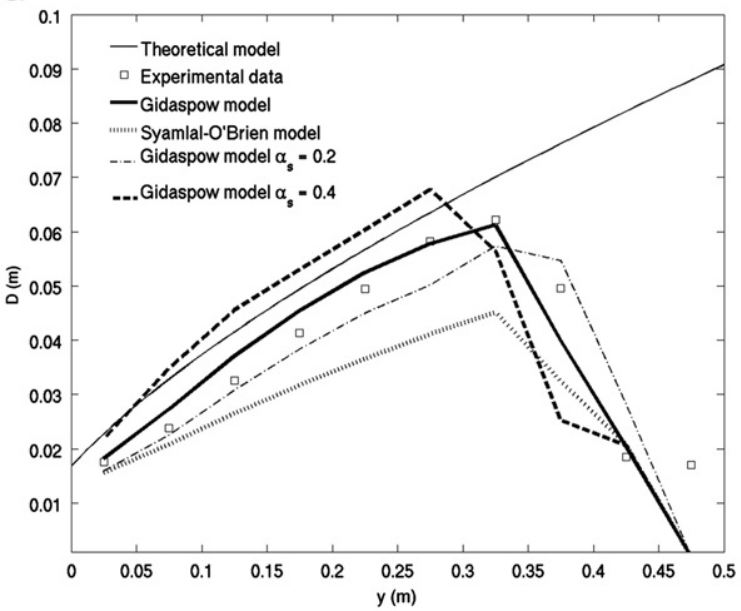

b

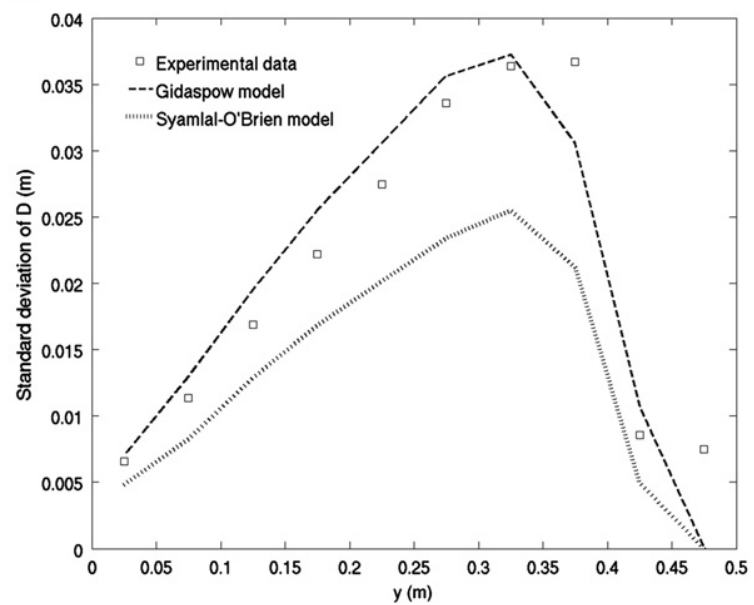

Fig. 9. Mean bubble diameter as a function of height from the distributor, $y$, obtained from simulation and experimental data (a) and its standard deviation (b).

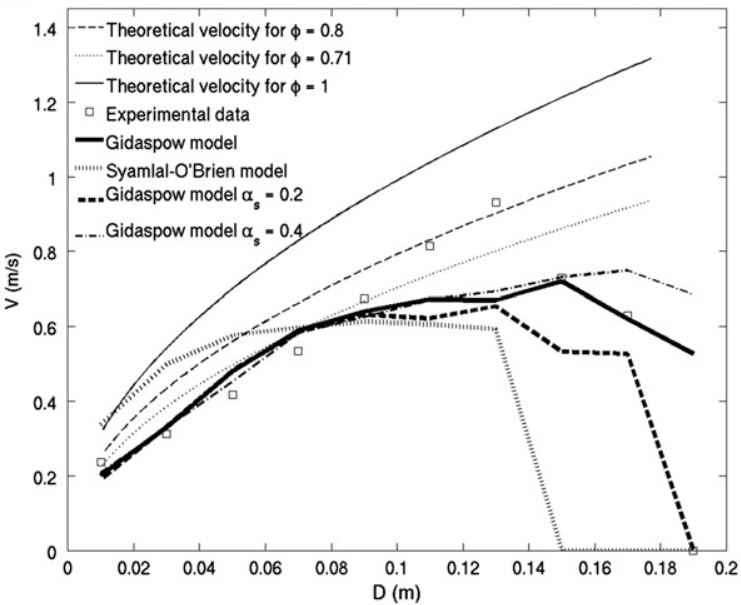

b

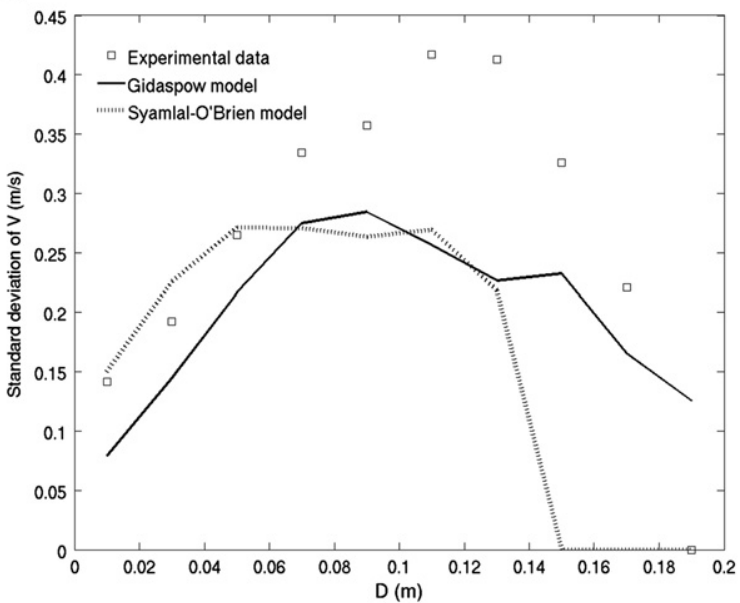

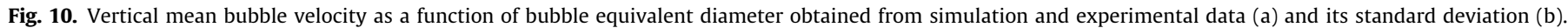

The standard deviation of the instantaneous values of the bubble diameter and velocity in Figs. 9b and 10b characterize the dispersion from the mean values.

As Fig. 9 illustrates, the Gidaspow's drag model yields the bubble growth with height in terms of both mean and standard deviation values, that most closely resembles the experimental data. In particular, the mean bubble diameter of the simulation with this drag model is slightly smaller but in acceptable agree ment with the theoretical model of Shen et al. (2004), Eq. (9), except at distances greater than the static height of the bed surface, $y 0.30 \mathrm{~m}$, where the theoretical model is not applicable. This level of similarity between model and simulation for mean bubble diameter profile along the bed height is also observed in other previous publications (see, for instance, Van Wachem et al., 1998; Patil et al., 2005) The experimental data of bubble diameter are inside the simulation data dispersion shown in Fig. 9b. The standard deviation of the simulated bubble diameter is remark able close to the experimental one. However, the experimental data do not fit the theoretical model as closely as the numerical results. The apparently more randomly behaviour of the experi mental results due to the lower number of bubbles taken from the images may be the reason of this discrepancy. It is symptomatic that both experiments and simulation predict larger bubble diameter in a similar degree near the bed surface, indicating that Eq. (9) could be adjusted and extended to this complex region of the bed. However, more experimental data would be advisable to support this improvement.

Fig. 9a also indicates that the drag model of Syamlal \& O'Brien tends to slightly underestimate the bubble growth from experi ments and from the model of Shen et al. (2004), when applied to the operative conditions studied in the present work. However, according to Fig. 10a, but this is not as clear for the standard deviation for the bubble velocity shown in Fig. 10b, the drag model of Syamlal \& O'Brien confers for relatively small bubbles, $D_{b} \leq 0.04 \mathrm{~m}$, the velocity dependence on bubble diameter most fitted to the classical dependence of Harrison and Davidson, Eq. (10) for $\varphi=0.71$. This seems to be facilitated by an under prediction of bubble velocity at low heights. The simulation based on Gidaspow drag model leads to the closest results to the experimental data in Fig. 10a. As a general trend, it can be said that the theoretical model for isolated bubbles due to Eq. (10) with $\varphi=0.71$, overpredicts the bubble velocity of the present experiments in the range of medium sized and large bubbles. Notice that the model of bubble growth used in this study 
assumes that bubbles are not close to the eruption stage, but large bubbles in Fig. 10a presumably are placed near the bed surface as Fig. 9a shows. This would explain the systematic deviation of the simulation results when $D_{b} \leq 0.09 \mathrm{~m}$.

In Figs. 9a and 10a results obtained for different bubble threshold, 0.2 and 0.4 are also included. The bubble diameter varies accordingly with this value. Obviously, larger bubbles are obtained when this value is 0.4 and smaller bubbles when the value is 0.2 , but both results are in accordance with the theore tical model. Paying attention to the bubble velocity, no differences can be seen in the range where data are reliable, this range covers bubble diameters up to $0.07 \mathrm{~m}$ placed up to $0.3 \mathrm{~m}$ above the distributor, which is the static bed height. In general, the value of 0.3 for bubble threshold provides the results most similar to experiments and the theoretical model.

\subsection{Air throughflow}

The distribution mechanisms of the air flow in the studied fluidized bed can be analysed to explain the encountered differ ences in dense phase velocities between the experiments and the simulation. As presented in Shen et al. (2004), three are the main distribution channels of air in the bed: the transport of air contained in bubbles, the air percolating between particles (i.e. minimum fluidisation air), and the air throughflow. The air throughflow is the excess of air that ascends bypassing pairs or groups of consecutive bubbles. It is calculated here using the conservation of mass balance of Shen et al. (2004)

$U_{t h}=U_{0} \quad\left(1 \quad \delta_{b}\right) U_{m f} \quad \delta_{b} U_{b}$

where $U_{t h}$ is the throughflow velocity, that is, the air throughflow per unit of distributor area, $U_{0}$ is the superficial air velocity, $U_{m f}$ is the minimum fluidisation velocity, and $U_{b}$ is the mean ascending velocity of the bubbles weighted with their frontal area:

$U_{b}=\frac{\sum_{i}^{N_{b}}{ }_{1} A_{b, i} v_{b, i}}{\sum_{i}^{N_{b}}{ }_{1} A_{b, i}}$

where $v_{b}$ and $A_{b}$ the ascending velocity and the frontal area, respectively, of a bubble, i.e. area on the images or simulation plane, and $N_{b}$ is the number of bubbles present in the studied region of the bed of frontal area $A_{f}$. In Eq. (11) $\delta_{b}$ is the fraction of the studied region that is occupied by bubbles:

$\delta_{b}=\frac{\sum_{i}^{N_{b}} A_{b, i}}{A_{f}}$

Using Eq. (11) the throughflow velocity has been calculated for the total sampling area of the bed, $A_{f}=w_{1} h_{1}$, and time averaged. Table 7 compares de simulation and experimental results. For the simulation, the reference case described in Table 1 has been selected, taking the same minimum fluidisation velocity than in the experiments, $U_{m f}=0.35 \mathrm{~m} / \mathrm{s}$.

According to the results of Table 7, the fractional area of the bed occupied by bubbles is only 3\% larger in the simulation than in the experiments. The visible bubble flow per distributor area, which is

Table 7

Simulation and experimental results for the visible flow and throughflow in the bed and bed expansion.

\begin{tabular}{lll}
\hline Variable & Simulation & Experimental \\
\hline$\delta_{b}$ & 0.122 & 0.118 \\
$U_{v i s}(\mathrm{~m} / \mathrm{s})$ & 0.0579 & 0.0410 \\
$U_{\text {th }}(\mathrm{m} / \mathrm{s})$ & 0.2466 & 0.2629 \\
Freeboard mean $y$-coordinate $(\mathrm{m})$ & 0.3771 & 0.3391 \\
\hline
\end{tabular}

calculated as $U_{v i s}=\delta_{b} U_{b}$, is an order of magnitude smaller than the other terms of Eq. (11), including the throughflow velocity $U_{t h}$. This agrees with the results presented by Shen et al. (2004). Besides, the visible bubble flow obtained from the simulation is more than $40 \%$ larger, and the throughflow is $6.6 \%$ smaller, than in the results from the experiments. This has important consequences in the analysis of the results: there is a slight underestimation of the throughflow velocity in the simulation compensated by an increase in visible flow. As the visible bubble flow is six or more times smaller than the throughflow, the slight underestimation of $U_{t h}$ generates an impor tant increase, in relative terms, in $U_{v i s}$ and also in $U_{b}$ provided $\delta_{b}$ remains nearly unchanged. Thus, using the weighted average of Eq. (12), the simulation performed is characterised by a larger $U_{v i s}$, which enhances the transport of dense phase compared to the experiments. However, this $40 \%$ increment of $U_{v i s}$ seems by itself unable to generate in most of the simulated bed a vertical flow of dense phase between 4 to more than 9 times superior than in experiments, as show in Table 1 . Therefore, other relevant causes in addition to the overprediction of $U_{b}$, may be behind the large values of the discrepancy $\delta_{r}$ in Table 1. One hypothesis is that some fraction of the air throughflow of Table 7 in the experimental fluidized bed is actually bypassing the dense phase, ascending just close to the wall surfaces, where the void fraction is large since the packing of particles close to the wall is not as efficient as in the bulk of the bed. If this attached to wall air flow is significant, it can reduce the throughflow crossing the bulk of the dense phase and, as a consequence, reduce the drag forces between gas and dense phase and hence decrement particle velocities in the experiments. Provided that in a real two dimensional fluidized bed the ratio surface area to volume is high (but not in the simulated bed), this may be a hypothesis that should not be disregarded. Another hypothesis is that the drag models between air and particles, lead to an overprediction of net forces on dense phase even if the throughflow velocity crossing the bulk of the bed is similar in simulation and experiments. Finally, due to the complex nonlinear nature of the bed hydrodynamics, it is also possible that the models accounting for frictional forces between particles may not reproduce the effective viscosity of the dense phase in regions involved in the transport of particles, such as the bubble wake. A dimensionless parameter can be defined for the visible bubble flow rate, as done by Hilligardt and Werther (1986).

$\psi=\frac{\text { visible bubble flow rate }}{\text { expected bubble flow }}=\frac{U_{v i s}}{U_{0} U_{m f}}$

This parameter indicates the fraction of the excess of flow actually carried by bubbles. The simulation and experimental results for $\psi$ are plotted in Fig. 11 together with two models. One of the models for $\psi$ has been taken from Hilligardt and Werther (1986):

$\psi=0.26$ for $y<0.275 \mathrm{~m}$

$\psi=0.35\left(\frac{y}{W}\right)^{0.5} \quad$ for $\quad y \geq 0.275 \mathrm{~m}$

where $y$ is the distance above de distributor and $W$ is the width of the bed.

The other model used is from Johnsson et al. (1991)

$\psi=f_{2}\left(y+4 \sqrt{ } A_{0}\right)^{0.4}$

where

$f_{2}=\left(0.26+0.70 \exp \left(3.3 d_{p} 10^{3}\right)\right)\left(0.15+\left(\begin{array}{ll}u & u_{m f}\end{array}\right)\right)^{-0.33}$

where $d_{p}$ is the particle diameter and $A_{0}$ is the area of the distributor per orifice.

As expected, the fraction of visible flow $\psi$ is higher in the simulation than in the experimental results, since Eq. (14) is proportional to $U_{v i s}$. Apart from the differences between the experimental and the simulation results, the model from 
Hilligartd and Werther (1986), gives a value of $\psi$ constant for the range of vertical position studied (i.e. from the bottom of the bed till the static bed height), and does not follow the growth experienced by the simulation and the experimental data. This can be explained by the fact that the model from Hilligardt and Werther (1986), was developed for a three dimensional fluidized bed. Besides, Eq. (15) has been selected for Geldart D particles since the model for Geldart B leads to $\psi=0.65$. On the other hand, the model of Johnsson et al. (1991), provides a growth of the fraction of visible flow that seems to be more representative of the two dimensional bed studied. Nevertheless, Eq. (16) does not tend to zero close to the distributor in contrast to the simulation and the experimental results presented in Fig. 11

The last part of this section compares the bed expansion obtained from the two fluid simulation (Gidaspow model) and

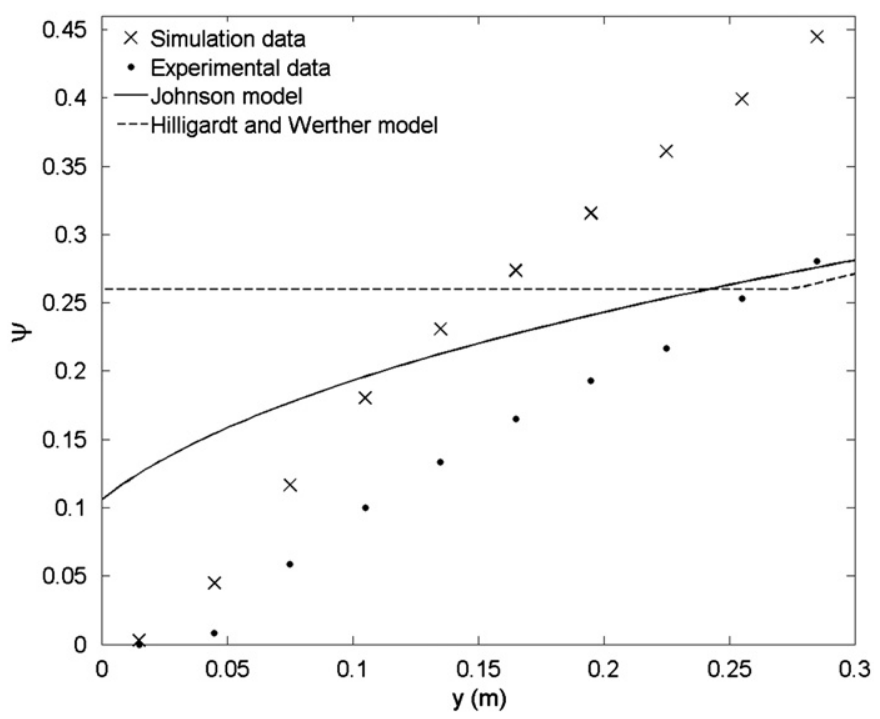

Fig. 11. Fraction of visible bubble flow. the experimental data. Results on this regard are included on Table 7. The bed expansion has been calculated with the mean $y$ coordinate of the freeboard (i.e. transversally averaged height of the bed surface), which has been time averaged over the same number of snapshots utilised for the calculation of bubble diameter and velocity in the previous section. The resulting time averaged mean $y$ coordinate of the freeboard is 0.3771 and $0.3391 \mathrm{~m}$ for the simulation and experimental results, respectively. As the visible flow in the simulation results is higher than in the experiments, more bubbles appear in the simulation and, therefore, the bed expansion has to be higher than in the real bed tested.

\subsection{Instantaneous interaction between bubble and dense phase}

A comparison of the instantaneous behaviour of dense phase surrounding bubbles can be valuable in order to explain the differences encountered between the simulation and experimen tal results. Fig. 12 shows the relative vectors of two coalescent bubbles for the simulation and experimental results superim posed to the instantaneous dense phase concentration maps and the images of particles, respectively.

Both simulation and experiments reveal the same physical interaction between bubbles and dense phase: downward motion of particles at the sides of the bubble, high particle velocity in the wake of the leading bubble, and deformation of the trailing bubble in the wake of the leading one.

Table 8 compares some hydrodynamics values of the two bubbles depicted in Fig. 12. Obviously, it was not possible to capture two identical pairs of coalescent bubbles in the experi ments and the simulation. In fact the pair of bubbles from the simulation is nearly two times bigger than the pair selected from the experimental images.

Fig. 13a depicts the streamlines of the dense phase velocity of the two coalescent bubbles obtained from experiments, Fig. 12b. In Fig. 13a, the image of particles of this part of the bed has been superimposed to the streamlines, being the bubbles the dark areas of the image. Also, the streamlines for the dense phase velocity of a

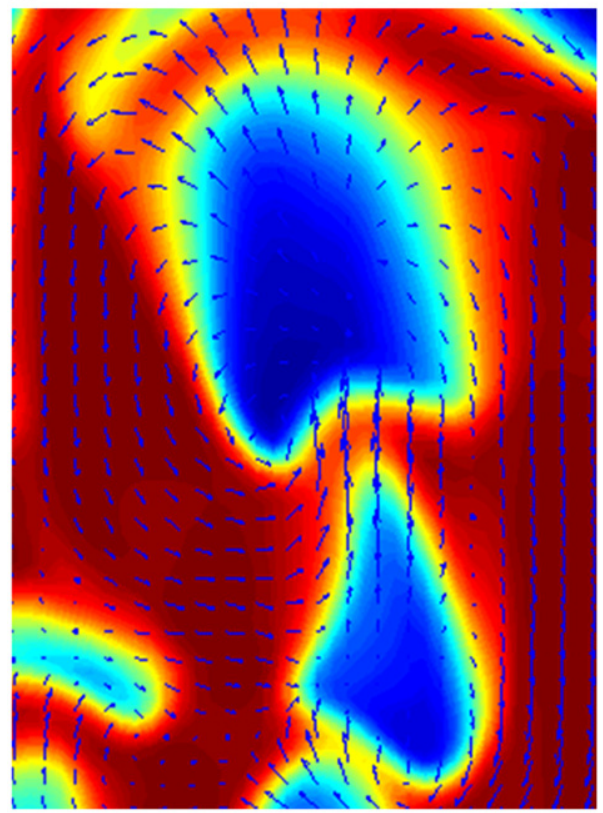

b

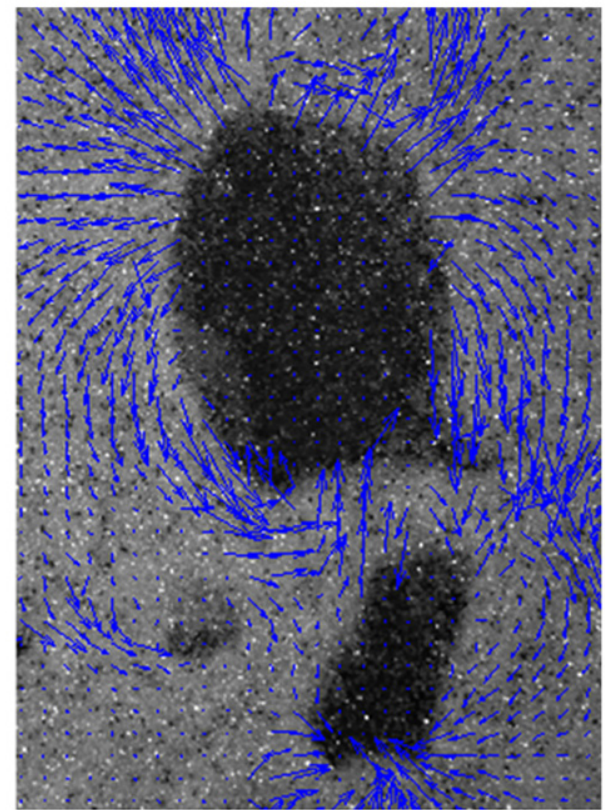

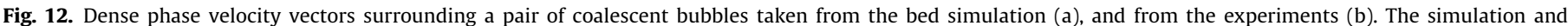
experimental results are, respectively, shown superimposed to their particle volume fraction map and image of particles. 
the bubbles in Fig. 12a, have been calculated for the simulation results and presented in Fig. 13b. In order to compare the simulation and experimental results in the same conditions, in Fig. 13a and b, the streamline calculation procedure described in Appendix B fills the interior of the bubbles with null dense phase velocity, since no information in PIV is obtained there. As explained in the Appendix B, the method of streamline calculation ensures soft curvature stream lines inside regions of unknown particle velocity. The actual streamlines of the falling particles inside bubbles is also expected to follow soft trajectories (i.e. parabolic trajectories). To verify this assumption, Fig. 13c presents the streamlines of the same simulated bubbles of Fig. 13b but now using all the velocity information of dense phase, that is, including the velocities inside bubbles. Note that no significant differences between Fig. 13b and c appear. Thus, it is assumed that the streamlines of Fig. 13a are weakly affected by the computing artefacts related to the lack of experimental velocity data inside bubbles.

Those pair of bubbles have been compared with the theoretical model developed by Toei and Matsumo (1967) for vertically aligned bubbles, that correlates its dimensionless position $H^{*}=H / H_{0, \min }$ versus its dimensionless time $T=\left(v_{b, L} t\right) / H_{0, \min }$, where $H$ corresponds with the location of the top of each bubble, $H_{0, \min }$ is the distance between the top of the leading bubble and the bottom of its wake, $v_{b, L}$ is the vertical velocity of the leading bubble, and $t$ is the time.

Table 8

Hydrodynamics related to two coalescent bubbles.

\begin{tabular}{lll}
\hline Parameter & Simulation & Experimental \\
\hline Leading bubble diameter $(\mathrm{m})$ & 0.0984 & 0.0585 \\
Leading bubble ascending velocity $(\mathrm{m} / \mathrm{s})$ & 0.924 & 0.413 \\
Leading bubble gas velocity $(\mathrm{m} / \mathrm{s})$ & 3.46 & - \\
Trailing bubble diameter $(\mathrm{m})$ & 0.0639 & 0.0310 \\
Trailing bubble ascending velocity $(\mathrm{m} / \mathrm{s})$ & 0.6 & 0.469 \\
Trailing bubble gas vertical velocity $(\mathrm{m} / \mathrm{s})$ & 3.38 & - \\
Particles downflow velocity $(\mathrm{m} / \mathrm{s})$ & -0.401 & -0.126 \\
Leading bubble wake velocity $(\mathrm{m} / \mathrm{s})$ & 1.20 & 0.202 \\
\hline
\end{tabular}

For the simulation results presented in Table 8 , the dimensionless position takes values of $H_{L}=3.05$ and $H_{T r}=1.65$ for the leading bubble and the trailing one, respectively, that lead to an increment of dimensionless position $\Delta H=H_{L} \quad H_{T r} \approx 1.4$. This value $H_{L}=3.05$ cor responds to a dimensionless time $T \approx 1.5$ and with $\Delta H_{\text {Model }} \approx 1.25$ according to the experimental correlation plot shown in Toei and Matsumo (1967). Regarding the experimental results of Table 8, the dimensionless positions are $H_{L}=3.43$ and $H_{T r}=2.12$, that is $\Delta H=1.31$. Using $H_{L}=3.43$ in the correlation of Toei and Matsumo (1967), a dimensionless time of $T \approx 1.8$ and an increment of dimensionless position $\Delta H_{\text {Model }}=1.13$ is obtained. Therefore, the experimental result for $\Delta H$ is $16 \%$ greater than the corresponding model prediction $\Delta H_{\text {Model }}$. Similarly, the numerical result for $\Delta H$ is $12 \%$ superior than $\Delta H_{\text {Model }}$. That means that both simulation and experimental results experience the same level of agreement with the bubble coalescence model presented by Toei and Matsumo (1967). As $1<T<2.5$ in for the simulation and experimental results, the coalescent bubbles of Table 8 are not out of the range of interaction and coalescence of vertically aligned bubbles shown in Toei and Matsumo (1967).

The same analysis has been done for a simulated and experi mental bubble as much isolated as the chaotic behaviour of the bed admit. The main hydrodynamics parameters of the selected semi isolated bubbles are included in Table 9, and Fig. 14 presents their streamlines calculated in the same way than in Fig. 12. In general, the streamlines from both simulation (Fig. 14b) and experiments (Fig. 14a) are qualitatively similar, and negligible

Table 9

Hydrodynamics parameters of semi-isolated bubbles.

\begin{tabular}{lcc}
\hline Parameter & Simulation & Experimental \\
\hline Bubble diameter $(\mathrm{m})$ & 0.0893 & 0.0680 \\
Bubble ascending velocity $(\mathrm{m} / \mathrm{s})$ & 0.611 & 0.266 \\
Bubble gas velocity $(\mathrm{m} / \mathrm{s})$ & 1.71 & - \\
Downflow velocity $(\mathrm{m} / \mathrm{s})$ & -0.402 & -0.174 \\
Wake velocity $(\mathrm{m} / \mathrm{s})$ & 0.565 & 0.245 \\
$\varphi \quad \frac{v_{b}}{\sqrt{g D_{\text {eq }}}}$ & 0.653 & 0.326 \\
\hline
\end{tabular}

a

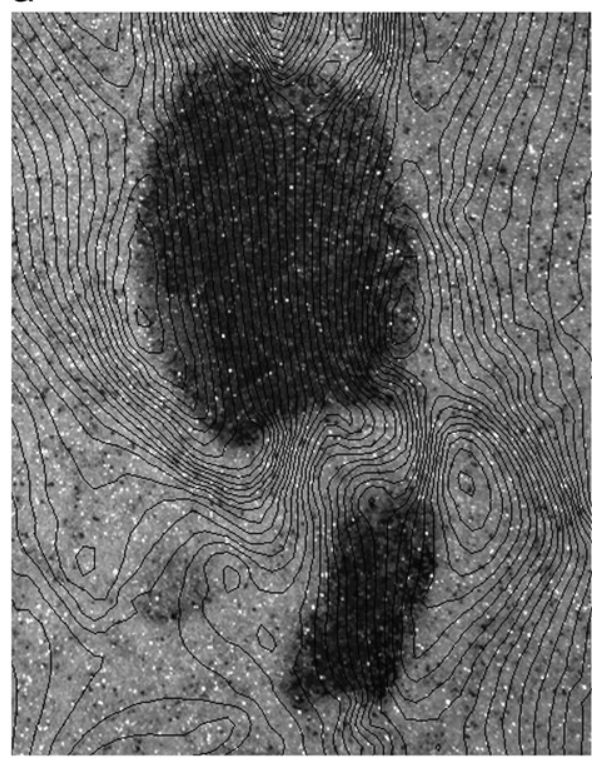

b

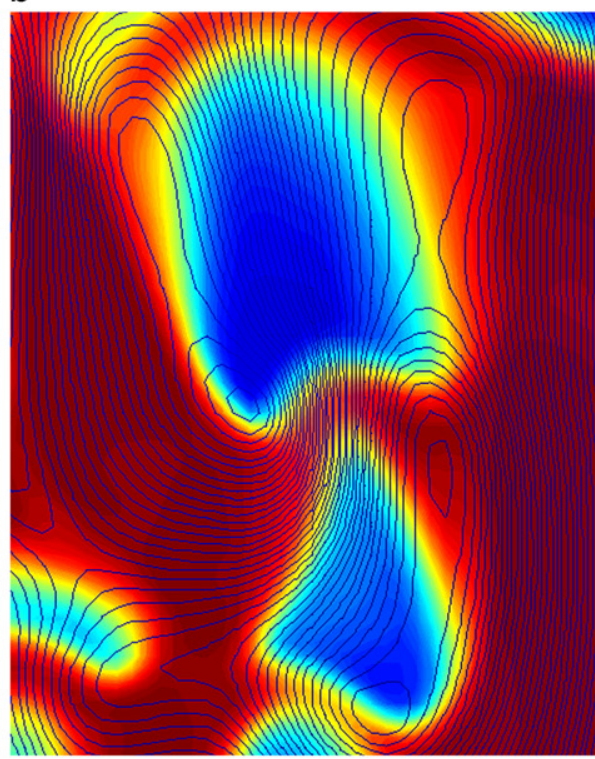

C

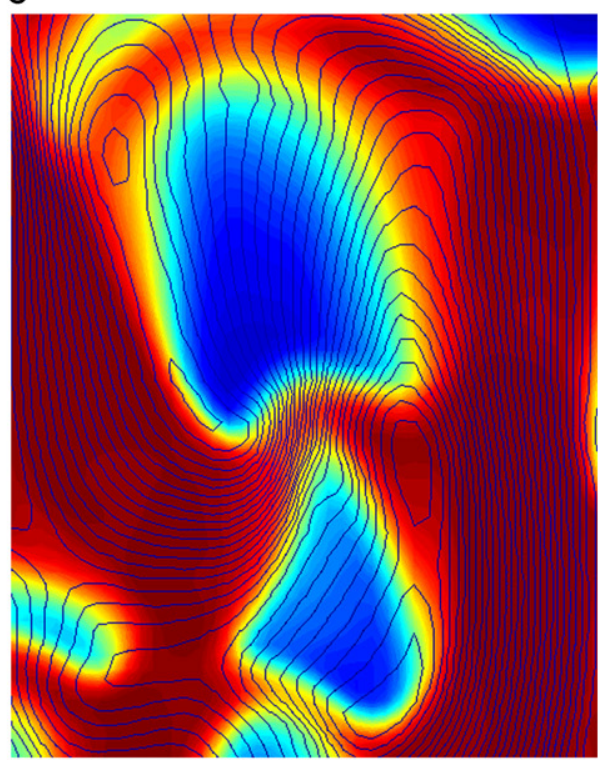

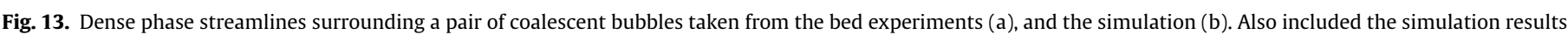

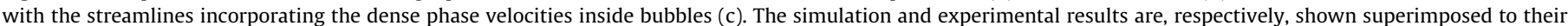
particle volume fraction map and image of particles. 
a

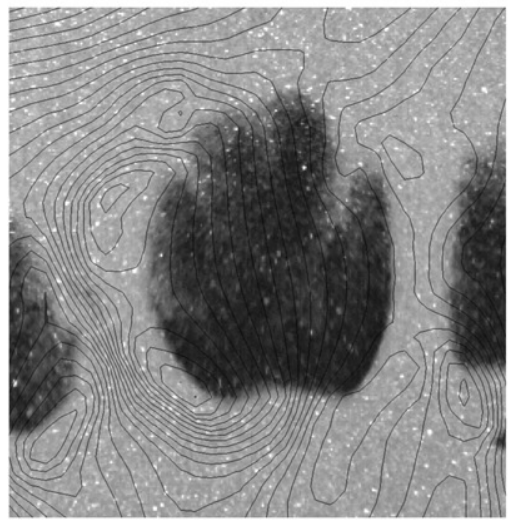

b

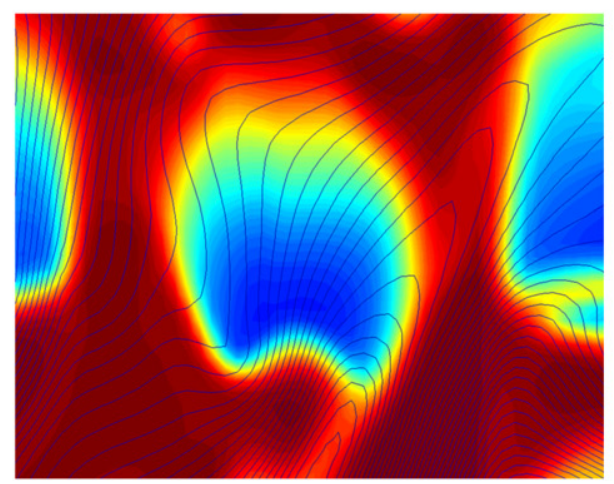

C

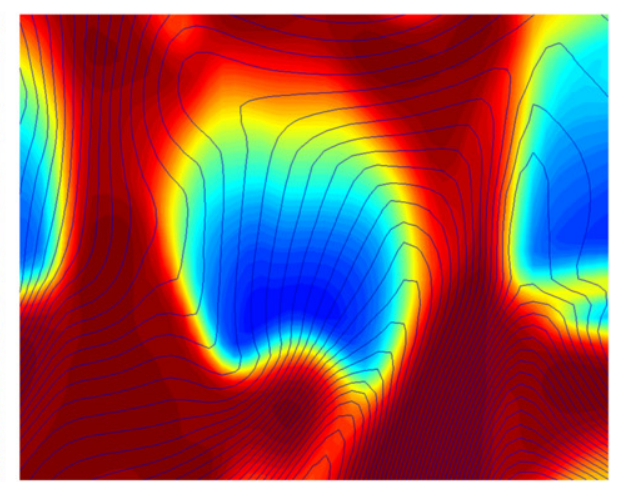

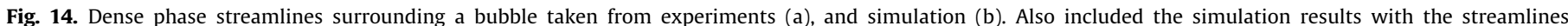

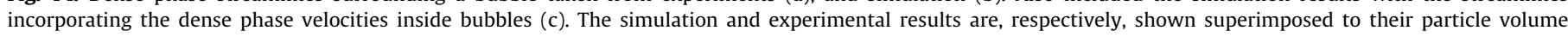
fraction map and image of particles.

differences appear between the simulation results for the stream lines calculated without and with the particle velocities in the bubbles (Fig. 14c).

The velocity coefficient $\varphi$ in Table 9 has been calculated with the instantaneous values of bubble diameter and velocity. Although the experimental and the simulation values obtained for $\varphi$ are of the same order, the experimental results in Table 9 present a value clearly far from the well known range $\varphi=0.71 \quad 1$. However, the instantaneous values of $\varphi$ are not necessarily representative of mean bubble conditions, and can be merely attributed to the stochastic behaviour of bubbling beds.

The bubble gas velocities presented in Tables 8 and 9 corre spond to the vertical velocity of the air at the centre of each bubble. Obviously, the gas velocity cannot be obtained from the experimental results because only the dense phase velocity is measured. It can be verified that the gas inside the bubble is much faster than the bubble rising velocity. The difference between the bubble gas velocity and the bubble velocity constitutes the throughflow, reaching in this particular bubble a value around $1.1 \mathrm{~m} / \mathrm{s}$, that is 2.9 times the minimum fluidisation velocity $U_{m f}$. That figure of throughflow is closer to the predictions of the Davidson theory for three dimensional bubbles $\left(3 U_{m f}\right)$ than for two dimensional ones $\left(2 U_{m f}\right)$ (Davidson and Harrison, 1963). For the case of coalescent bubbles, Table 8 , the throughflow velocity elevates to 2.54 and $2.78 \mathrm{~m} / \mathrm{s}$ for the rear and trailing bubble. The throughflow of air is the responsible of the acceleration of particles between coalescent bubbles. As Table 8 shows, the particle velocity between the coalescent bubbles (i.e. wake of the leading bubble) is nearly six times superior in the simulation than in the PIV experiments, but the diameter of the simulated bubbles selected in Table 8 is less than twice that of the experiments. Note also that the downflow velocity of particles at both sides of the leading bubble (Table 8 ) in the case of the simulation is less than 3.2 times intense than in the experiments. Assuming that the solids velocity is proportional to the coalescent bubbles velocity and their level of approximation (proportional to $T$ ), the air between the coalescent bubbles selected from the simulation in Table 8 would be more efficient in driving particles than in the coalescent bubbles selected from experiments. An overprediction of the air drag due to models tested can be an important cause of the large particle velocity encountered between simulated bubbles compared to PIV measurements, since the drag force of air is the main factor participating in the raising forces on a particle. From Table 9, it is also noticeable that the wake velocity as well as the particle downflow velocity of the simulated bubble is around 2.3 times larger than the experimen tally measured bubble. However, the velocity of the simulated bubble is also 2.3 larger than the experimental one, which explains the reason of that difference in the wake velocity of the semi isolated bubbles selected in Table 9.

Other kinds of bubbles morphologies (splitting, erupting and wall attached bubbles) may be analysed but have not been included here for brevity reasons.

\section{Summary and conclusions}

Two major kinds of information have been analysed in the present study aimed to the comparison of Eulerian Eulerian two fluid simulation and experimental results in a two dimensional gas solid fluidized bed: bubble hydrodynamics, and dense phase (i.e. particle phase) velocity. In particular, in contrast to previous comparison studies usually focused on bubble behaviour and dense phase distribution, the present work examines and com pares not only the bubble hydrodynamics and dense phase probability within the bed, but also the time averaged vertical and horizontal component of the dense phase velocity, the air throughflow and the instantaneous interaction between bubbles and dense phase.

To increase generality of results, the two fluid simulations presented in this work were conducted using two different and well known closure models for the gas particle interaction. For verifying the simulation results, an experimental bed of $0.005 \mathrm{~m}$ thickness was measured here to ensure the two dimensionality of the bed dynamics. The experimental data from this bed concern ing bubble behaviour and dense phase distribution were obtained in the present work through non intrusive techniques based on the digital analysis of a long temporal sequence of images. In harmony with previous studies present in literature, the two fluid models tested here were able to qualitatively predict the bubble growth and velocity as well as the bubble probability distribution within the bed. Furthermore, an acceptable level of quantitative agreement between two fluid simulation and experiments is confirmed in this study regarding bubble diameter and velocity in a two dimensional bed. The similitude encountered between the simulated and experimental standard deviations of bubble diameter and velocity are also noticeable. The results of the present work reveals that, of the two drag models tested, the model of Gidaspow (1994) provides the best approach to the experiments and to simplified models based on the two phase 
theory of fluidized beds. Exception of this is the bubble prob ability profiles in the studied two dimensional bed, which seem to be best predicted using the Syamlal \& O'Brien drag model (Syamlal and O'Brien, 1987). Interestingly, both drags models, Gidaspow and Syamlal \& O'Brien, provide in this work a better bubble distribution (i.e. more homogeneous bubble probability maps) than the experimental bed.

Thanks to the simulation results, the present work clearly proves that the dense phase probability can be used as a good approximation of time averaged particle volume fraction in a bubbling bed. In the simulations, since the transition of bubble phase too dense phase is not as sharp as in experiments, a bubble detection threshold equal to the arithmetic mean between the maximum and minimum particle concentration is recommended. This important fact, which seems to be obviated by other previous publications, indicate that simple digital image techniques can be used to obtain satisfactory estimations of time averaged particle concentration in a two dimensional bed.

The main novelty of the present work is the quantitative comparison between simulations and experiments performed for the vertical as well as the horizontal component of the time averaged dense phase velocity in the whole plane of the two dimensional bed of reduced thickness. Regarding the experimental velocity of the dense phase, it was extracted in this work from the images of the real bed thanks to the application of a multigrid particle image velocimetry (PIV) technique. The resulting experi mental velocities of particles obtained in this work are coherent with other PIV measurements of bubbling beds of small thickness reported in literature. The comparison of time averaged velocity of dense phase from simulation and experiments was done here through the use of horizontal profiles of velocity as well as the definition of a discrepancy factor, which allows a quantitative comparison of dense phase velocities not conducted before in bubbling fluidized beds studies. Additionally, a global analysis of the dense phase velocity field from both simulation and experi ment data was done in the present study with the aid of streamlines maps calculated using an original and robust method developed ad hoc that can be applicable to simulation and noisy experimental data. Surprisingly, and despite the above described similarities in bubble diameter and velocity, the results of the present work indicate that the time averaged velocities of the particle phase in the simulated bed are nearly one order of magnitude larger than the velocities obtained from PIV experiments. Therefore, the fine agreement in bubble behaviour found between simulation and experiments does not ensure the same level of agreement in dense phase velocities in the framework of two dimensional beds. This significance result, not reported previously in literature, occurs for the two well known drag models tested, indicating that in Eulerian Eulerian two fluid simulations the beha viour of bubble velocity and diameter is relatively uncoupled from, or insensitive to, the resulting dense phase velocity. Since the superficial air velocities in the simulation and in the experimental bed have been set to similar values in this study, three principal causes of such a high discrepancy can be postulated: (1) the possible overestimative nature of the particle drag models in reproducing the net forces of the gas on a particle in a two dimensional regime; (2) the effects of particle wall friction that may be present at the frontal and back wall of a real quasi two dimensional bed, and (3) the influence of the higher porosity near the wall in a real fluidized bed, which may bypass a significant fraction of the air near the walls of a two dimensional bed and be the cause of the reduced values of bubble visible flow (the driver mechanism of particles) found in the experiments of this work compared to the performed simulations. Further research involving perhaps different experi ments and operative conditions are needed to discriminate the relative influence of the postulated causes of the discrepancy in dense phase velocity.

\section{Nomenclature}

$\bar{C} \quad$ proportion of time that a point is occupied by solids

$\bar{B} \quad$ proportion of time that a point is occupied by bubbles

$C_{D} \quad$ drag coefficient, dimensionless

$d_{i} \quad$ diameter, $\mathrm{m}$

$D_{b} \quad$ bubble diameter, $m$

$e_{s s} \quad$ restitution coefficient, dimensionless

$g_{0 s s} \quad$ radial distribution coefficient, dimensionless

$K_{g s} \quad$ gas/solid momentum exchange coefficient, $\mathrm{kg} /\left(\mathrm{m}^{3} \mathrm{~s}\right)$

$p \quad$ pressure, $\mathrm{Pa}$

$\vec{g} \quad$ acceleration due to gravity, $9.8 \mathrm{~m} / \mathrm{s}^{2}$

$k_{\Theta s} \quad$ diffusion coefficient for granular energy, $\mathrm{kg} /\left(\mathrm{m}^{3} \mathrm{~s}\right)$

$v_{i} \quad$ velocity, $\mathrm{m} / \mathrm{s}$

$h$ bed height, $\mathrm{m}$

$\dot{m}_{p} \quad$ particle mass flow rate, $\mathrm{kg} / \mathrm{s}$

$v_{b} \quad$ bubble vertical velocity, $\mathrm{m} / \mathrm{s}$

$\overrightarrow{v_{i}} \quad$ velocity vector, $\mathrm{m} / \mathrm{s}$

$\vec{V} \quad$ mean particle velocity, $\mathrm{m} / \mathrm{s}$

$t$ time, $s$

$T$ dimensionless time

$H \quad$ vertical location of the bubble top, $m$

$U_{0} \quad$ superficial velocity, $\mathrm{m} / \mathrm{s}$

$U_{t h} \quad$ throughflow velocity, $\mathrm{m} / \mathrm{s}$

$U_{v i s} \quad$ visible flow

$U_{b} \quad$ mean vertical bubble velocity, $\mathrm{m} / \mathrm{s}$

$x \quad$ horizontal position, $\mathrm{m}$

$y \quad$ vertical position, $\mathrm{m}$

$Z$ bed thickness, $m$

\section{Greek letters}

$\begin{array}{ll}\alpha_{i} & \text { volume fraction, dimensionless } \\ \delta & \text { root mean square discrepancy } \\ \delta_{r} & \text { relative discrepancy } \\ \delta_{b} & \text { fractional area occupied by bubbles } \\ \Theta_{i} & \text { granular temperature, } \mathrm{m}^{2} / \mathrm{s}^{2} \\ \lambda & \text { bubble growth coefficient } \\ \mu_{i} & \text { shear viscosity, } \mathrm{kg} / \mathrm{s} \mathrm{m} \\ \rho_{i} & \text { density, } \mathrm{kg} / \mathrm{m}^{3} \\ i & \text { stress tensor, Pa } \\ \varphi & \text { bubble velocity coefficient } \\ \psi & \text { fraction of visible flow }\end{array}$

\section{Subscripts}

$\begin{array}{ll}g & \text { gas } \\ i & \text { general index } \\ s & \text { solids } \\ m f & \text { minimum fluidisation } \\ L & \text { leading bubble } \\ R C & \text { recirculation centre } \\ T r & \text { trailing bubble }\end{array}$

\section{Acknowledgements}

This work has been partially funded by the Spanish Govern ment (Project DPI2009 10518) and the Autonomous Community of Madrid (Project S2009/ENE 1660). Their supports are greatly appreciated. 


\section{Appendix A. Closure expressions}

\section{A.1. Particle drag coefficient}

The model of Gidaspow used for the drag function between particles and gas phase in the simulation is (Gidaspow, 1994):

$K_{g s}=\frac{3}{4} C_{D} \frac{\alpha_{s} \alpha_{g} \rho_{g}\left|\overrightarrow{v_{s}} \quad \overrightarrow{v_{g}}\right|}{d_{s}} \alpha_{g}^{-2.65}$ for $\alpha_{g}>0.8$

$K_{g s}=150 \frac{\alpha_{s}^{2} \mu_{g}}{\alpha_{g} d_{s}{ }^{2}}+1.75 \frac{\alpha_{s} \rho_{g}\left|\overrightarrow{v_{s}} \overrightarrow{v_{g}}\right|}{d_{s}}$ for $\alpha_{g} \leq 0.8$

where the drag coefficient is defined as

$C_{D}=\frac{24}{\alpha_{g} \operatorname{Re}_{s}}\left[1+0.15\left(\alpha_{g} \operatorname{Re}_{s}\right)^{0.687}\right]$

with $R e_{s}=\frac{\rho_{g} d_{s}\left|\overrightarrow{v_{s}} \overrightarrow{v_{g}}\right|}{\mu_{g}}$

The model of Syamlal \& O'Brien (Syamlal and O'Brien, 1989) for the drag function is

$K_{g s}=\frac{3 \alpha_{s} \alpha_{g} \rho_{g}}{4 v_{r, s}^{2} d_{s}} C_{D}\left(\frac{R e_{s}}{v_{r, s}}\right)\left|\overrightarrow{v_{s}} \quad \overrightarrow{v_{g}}\right|$

where the drag coefficient is defined as

$C_{D}=\left(0.63+\frac{4.8}{\sqrt{ } \operatorname{Re}_{s} / v_{r, s}}\right)$

with $R e_{s}=\frac{\rho_{g} d_{s}\left|\overrightarrow{v_{s}} \overrightarrow{v_{g}}\right|}{\mu_{\mathrm{g}}}$

where

$v_{r, s}=0.5\left(A \quad 0.06 R e_{s}+\sqrt{ }\left(0.06 R e_{s}\right)^{2}+0.12 R e_{s}(2 B \quad A)+A^{2}\right)$

with $A=\alpha_{g}^{4.14} \quad B=\left\{\begin{array}{cc}0.8 \alpha_{g}^{1.28}, & \alpha_{g} \leq 0.85 \\ \alpha_{g}^{2.65}, & \alpha_{g}>0.85\end{array}\right.$

\section{A.2. Solids pressure}

The Solids pressure can be expressed as a function of the particle granular temperature (Lun et al., 1984)

$p_{s}=\alpha_{s} \rho_{s} \Theta_{s}+2 \rho_{s}\left(1+e_{s s}\right) \alpha_{s}^{2} g_{0, s s} \Theta$

where

$g_{0, s s}=\left[1\left(\frac{\alpha_{s}}{\alpha_{s, \max }}\right)^{1 / 3}\right]^{-1}$

with $\alpha_{s, \max }$ is the maximum packing limit.

\section{A.3. Solids stress tensor}

The solid phase stress tensor is defined as

$\overline{\bar{\tau}}_{s}=\alpha_{s} \mu_{s}\left(\nabla \overrightarrow{v_{s}}+\nabla{\overrightarrow{v_{s}}}^{T}\right)+\alpha_{s}\left(\lambda_{s} \frac{2}{3} \mu_{s}\right) \nabla \cdot \overrightarrow{v_{s}} \overline{\bar{I}}$

where solid shear viscosity is

$\mu_{s}=\mu_{s, c o l}+\mu_{s, f r}+\mu_{s, k i n}$

The model of Gidaspow for the kinetic viscosity (Gidaspow, 1994):

$\mu_{s, k i n}=\frac{10 \rho_{s} d_{s} \sqrt{ } \Theta \pi}{96 \alpha_{s}\left(1+e_{s s}\right) g_{0 s s}}\left[1+\frac{4}{5} g_{0 s s} \alpha_{s}\left(1+e_{s s}\right)\right]^{2}$
The model of Syamlal O'Brien for the kinetic viscosity (Syamlal et al., 1993):

$\mu_{s, k i n}=\frac{\alpha_{s} \rho_{s} d_{s} \sqrt{ } \Theta \pi}{6\left(3 e_{s s}\right)}\left[1+\frac{2}{5}\left(1+e_{s s}\right)\left(3 e_{s s} 1\right) \alpha_{s} g_{0 s s}\right]$

The collisional viscosity (Gidaspow, 1994; Syamlal et al., 1993) is defined with

$\mu_{s, c o l}=\frac{4}{5} \alpha_{s} \rho_{s} d_{s} g_{0, s s}\left(1+e_{s s}\right)\left(\frac{\Theta}{\pi}\right)^{1 / 2}$

And the frictional viscosity (Schaeffer, 1987):

$\mu_{s, f r}=\frac{p_{s} \sin \phi}{2 \sqrt{ } I_{2 D}}$

\section{A.4. Diffusion of granular temperature}

The model of Gidaspow for the diffusion coefficient of granular temperature (Gidaspow, 1994)

$$
\begin{aligned}
k_{\Theta}= & \frac{150 \rho_{s} d_{s} \sqrt{ } \Theta \pi}{384\left(1+e_{s s}\right) g_{0 s s}}\left[1+\frac{6}{5} \alpha_{s} g_{0 s s}\left(1+e_{s s}\right)\right]^{2} \\
& +2 \rho_{s} d_{s} \alpha_{s}^{2} g_{0 s s}\left(1+e_{s s}\right) \sqrt{\frac{\Theta_{s}}{\pi}}
\end{aligned}
$$

The model of Syamlal \& O'Brien (Syamlal et al., 1993) for the diffusion coefficient of granular temperature:

$k_{\Theta}=\frac{15 \rho_{s} d_{s} \alpha_{s} \sqrt{ } \Theta \pi}{4(4133 \eta)}\left[1+\frac{12}{5} \eta^{2}(4 \eta \quad 3) \alpha_{s} g_{0 s s}+\frac{16}{15 \pi}\left(\begin{array}{ll}41 & 33 \eta\end{array}\right) \eta \alpha_{s} g_{0 s s}\right]$

with $\eta=\frac{1}{2}\left(1+e_{s s}\right)$

where $\varphi$ is the angle of internal friction, and $I_{2 D}$ is the second invariant of the deviatory stress tensor.

\section{A.5. Collisional dissipation of energy}

Finally, the collisional dissipation of energy is modelled as (Lun et al., 1984):

$\gamma_{\Theta}=\frac{12\left(1 \quad e_{s s}^{2}\right) g_{0, s s}}{d_{s} \sqrt{ } \pi \rho_{s} \alpha_{s}^{2} \Theta^{3 / 2}}$

\section{Appendix B. Streamlines computation}

Given that the particulate phase, outside bubbles, in fluidized beds behaves like an incompressible fluid (Davidson and Harrison, 1963), it is possible to use a Poisson equation for the computation of the streamlines of the particulate phase in a two dimensional flow. This procedure is described in the following lines. Precaution in the interpretation of the streamlines should be taken for regions of the bed where the void fraction of particles varies greatly, such in the bubble interphase and bed surface, and can depart from the incompressibility condition. This can be easily deduced from the Eulerian equations of the particulate phase (Eq. 2 and 4). However, these regions are narrow in size and the dissipative nature of the Poisson equation yields smooth stream lines, avoiding unrealistic oscillations or abrupt changes in the streamlines direction. For the same reason, the method is especially suitable for extracting the streamlines when the velocity field is affected by measurement noise.

In a two dimensional incompressible flow, the stream function $\psi$ is related to the horizontal and vertical components of the 
velocity, $u$ and $v$, respectively:

$\frac{\partial \psi}{\partial y}=u$

$\frac{\partial \psi}{\partial x}=v$

Introducing Eq. (B.1) and (B.2) into the vorticity definition

$\zeta=\frac{\partial v}{\partial x} \quad \frac{\partial u}{\partial y}$

the next Poisson equation for the stream function appears

$\frac{\partial^{2} \psi}{\partial x^{2}}+\frac{\partial^{2} \psi}{\partial y^{2}}=\zeta$

With the appropriate boundary conditions for $\psi$, Eq. (B.4) can be solved and the streamlines computed from the isovalue lines of the stream function.

In the present work, centred finite differences have been used for the discretization of the spatial derivatives in a rectangular mesh not necessarily homogeneous

$\zeta_{i, j} \approx \frac{v_{i+1, j} \quad v_{i-1, j}}{\Delta x_{i}+\Delta x_{i-1}} \quad \frac{u_{i, j+1} \quad u_{i, j-1}}{\Delta y_{j}+\Delta y_{j-1}}$

$\left.\frac{\partial^{2} \psi}{\partial x^{2}}\right|_{i, j} \approx \frac{2}{\Delta x_{i-1}+\Delta x_{i}}\left(\left.\left.\frac{\partial \psi}{\partial x}\right|_{i+(1 / 2), j} \frac{\partial \psi}{\partial x}\right|_{i-(1 / 2), j}\right)$

$\left.\frac{\partial^{2} \psi}{\partial y^{2}}\right|_{i, j} \approx \frac{2}{\Delta y_{j-1}+\Delta y_{j}}\left(\left.\left.\frac{\partial \psi}{\partial y}\right|_{i, j+(1 / 2)} \frac{\partial \psi}{\partial y}\right|_{i, j-(1 / 2)}\right)$

where the indices $(i, j)$ refer to the position $\left(x_{i}, y_{j}\right)$ of a node in the computational mesh, and $(i+1 / 2, j),(i 1 / 2, j)$, and so on, account for discretizations placed in intermediate positions between nodes; for example:

$\left.\frac{\partial \psi}{\partial x}\right|_{i+(1 / 2), j} \approx \frac{\psi_{i+1, j} \quad \psi_{i, j}}{\Delta x_{i}}$

$\left.\frac{\partial \psi}{\partial y}\right|_{i, j+(1 / 2)} \approx \frac{\psi_{i, j+1} \quad \psi_{i, j}}{\Delta y_{j}}$

Note that $\Delta x_{i}=x_{i+1} \quad x_{i}, \Delta x_{i} \quad{ }_{1}=x_{i} \quad x_{i} \quad 1, \Delta y_{j}=y_{j+1} \quad y_{j}$ and $\Delta y_{j} \quad{ }_{1}=y_{j} \quad y_{j} \quad 1$ are the horizontal and vertical separation of consecutive nodes. In a homogeneous mesh $\Delta x_{i}$ and $\Delta y_{j}$ are constant regardless the node position and the discretization becomes second order accurate. It is assumed that the dense phase does not penetrate the walls and the distributor. Therefore, these boundaries are defined by a single value of $\psi$, which is arbitrarily assigned to zero. The value of $\psi$ at the upper part of the bed, i.e. the free board, can be readily calculated integrating Eq. (B.2) (or its discretized version as in (B.5)) along horizontal direction.

With the described discretization of spatial derivatives, Eq. (B.4) can be expressed as

$(a+b+c+d) \psi_{i, j} \quad a \psi_{i+1, j} \quad b \psi_{i-1, j} \quad c \psi_{i, j+1} \quad d \psi_{i, j-1}=\zeta_{i, j}$

where,

$$
\begin{aligned}
& a=\frac{2}{\Delta x_{i}+\Delta x_{i-1}} \frac{1}{\Delta x_{i}} \\
& b=\frac{2}{\Delta x_{i}+\Delta x_{i-1}} \frac{1}{\Delta x_{i-1}} \\
& c=\frac{2}{\Delta y_{j}+\Delta y_{j-1}} \frac{1}{\Delta y_{j}} \\
& d=\frac{2}{\Delta y_{j}+\Delta y_{j-1}} \frac{1}{\Delta y_{j-1}}
\end{aligned}
$$

The expression (B.10) conforms a system of equations, each one accounting for a node point $(i, j)$, that can be solved with a standard matrix inversion procedure such as Gauss Seidel or SOR methods. The above simple approximations of derivatives are not computationally demanding and provide satisfactory results for the fluidized beds tested. Obviously more precise and sophisticated techniques can be used for the solution of Eq. (B.4).

\section{References}

Ahuja, G.H., Patwardhan, A.W., 2008. CFD and experimental studies hold-up distribution and circulation patterns in gas-solid fluidized beds. Chemical Engineering Journal 143, 147-160.

Almendros-Ibáñez, J.A., Sobrino, C., de Vega, M., Santana, D., 2006. A new model for ejected particle velocity from erupting bubbles in 2-D fluidized beds. Chemical Engineering Science 61, 5981-5990.

Basu, P., 2006. Combustion and Gasification in Fluidized Beds. CRC, Taylor \& Francis, Boca Raton.

Boemer, A., Qi, H., Renz, U., 1998. Verification of Eulerian simulation of spontaneous bubble formation in a fluidized bed. Chemical Engineering Science 53, 1835-1846.

Bokkers, G.A., Laverman, J.A., van Sint Annaland, M., Kuipers, J.A.M., 2006. Modelling of large-scale dense gas-solid bubbling fluidised beds using a novel discrete bubble model. Chemical Engineering Science 61, 5590-5602.

Bokkers, G.A., van Sint Annaland, M., Kuipers, J.A.M., 2004. Mixing and segregation in a bidisperse gas-solid fluidised bed: a numerical and experimental study. Powder Technology 140, 176-186.

Busciglio, A., Vella, G., Micale, G., Rizzuti, L., 2009. Analysis of the bubbling behaviour of 2D gas-solid fluidized beds. Part II. Comparison between experiments and numerical simulations via Digital Image Analysis Technique. Chemical Engineering Journal 148, 145-163.

Caicedo, G.R., Prieto Marqués, Juan J., Ruíz, Mónica García, Soler, Jesús Guardiola, 2003. A study on the behaviour of bubbles of a 2D gas-solid fluidized bed using digital image analysis. Chemical Engineering and Processing 42, 9-14.

Dan, S., Jianzhi, W., Huilin, L., Yunhua, Z., Juhui, C., Gidaspow, D., Ming, C., 2010. Numerical simulation of gas-particle flow with a second-order moment method in bubbling fluidized beds. Powder Technology 199, 213-225.

Darton, R.C., LaNauze, R.D., Davidson, J.F., Harrison, D., 1977. Bubble growth due to coalescence in fluidized beds. Transactions of the Institute Chemical Engineering $55,274-280$.

Davidson, J.R., Harrison, D., 1963. Fluidized Particles. Cambridge University Press.

Davidson, J.R., Harrison, D., 1971. Fluidization. Academic Press, New York.

Deen, N.G., van Sint Annaland, M., van der Hoef, M.A., Kuipers, J.A.M., 2007. Review of discrete particle modelling of fluidized beds. Chemical Engineering Science $62,28-44$.

Deza, M., Franka, N.P., Heindel, T.J., Battaglia, F., 2009. CFD modelling and X-ray imaging of biomass in a fluidized bed. Journal of Fluids Engineering, 131.

Fluent, 2006. Fluent 6.3 Documentation. FLUENT Inc. 〈http://fluent.com/ >.

Geldart, D., 1973. Types of gas fluidization. Powder Technology 7, 285-292.

Gidaspow, D., 1994. Multiphase Flow and Fluidization: Continuum and Kinetic Theory Descriptions. Academic Press, San Diego.

Goldschmidt, M.J.V., Kuipers, J.A., van Swaaij, W.P.M., 2001. Hydrodynamic modelling of dense gas-fluidised beds using the kinetic theory of granular flow: effect of coefficient of restitution on bed dynamics. Chemical Engineering Science 56, 571-578.

Grace, J.R., Taghipour, F., 2004. Verification and validation of CFD models and dynamic similarity for fluidized beds. Powder Technology 139, 99-110.

Hilligardt, K., Werther, J., 1986. Local bubble gas hold-up and expansion of gas/ solid fluidized beds. German Chemical Engineering 9, 215-221.

Hosseini, S.H., Ahmadi, G., Rahimi, R., Zivdar, M., Esfahany, M.N., 2010. CFD studies of solids hold-up distribution and circulation patterns in gas-solid fluidized beds. Powder Technology 200, 202-215.

Johnsson, F., Andersson, S., Leckner, B., 1991. Expansion of a freely bubbling fluidized bed. Powder Technology 68, 117-123.

Jung, J., Gidaspow, D., Gamwo, I.K., 2005. Measurement of two kinds of granular temperatures, stresses and dispersion in bubbling beds. Industrial and Chemical Engineering Research 44, 1329-1341.

Jung, J., Gidaspow, D., 2006. Bubble computation, granular temperatures, and Reynolds Stresses. Chemical Engineering Communications 193, 946-975.

Kunii, D., Levenspiel, O., 1991. Fluidization Engineering. Butterworth-Heinemann Series in Chemical Engineering.

Ladd, A.J.C., Verberg, R., 2001. Lattice-Boltzmann simulations of particle fluid suspensions. Journal of Statistical Physics 104, 1191-1251.

Laverman, J.A., Roghair, I., van Sint Annaland, M., Kuipers, H., 2008. Investigation into the hydrodynamics of gas-solid fluidized beds using particle image velocimetry coupled with digital image analysis. Canadian Journal Chemical Engineering 86, 523-535.

Li, T., Pougatch, K., Salcudean, M., Grecov, D., 2009. Numerical simulation of single and multiple gas jets in bubbling fluidized beds. Chemical Engineering Science 64, 4884-4898.

Li, T., Grace, J., Bi, X., 2010. Study of wall boundary condition in numerical simulations of bubbling fluidized beds. Powder Technology 203, 447-457. 
Lin, J.S., Chen, M.M., Chao, B.T., 1985. A novel radioactive particle tracking facility for measurement of solids motion in gas fluidized beds. AIChE Journal 31, 465-473.

Lun, C.K.K., Savage, S.B., Jeffrey, D.J., Chepurniy, N., 1984. Kinetic theories for granular flow: inelastic particles in Couette flow and slightly inelastic particles in a general flow field. Journal of Fluid Mechanics 140, 223-256.

Lindborg, H., Lysberg, M., Jakobsen, H.A., 2007. Practical validation of the two-fluid model applied to dense gas-solid flows in fluidized beds. Chemical Engineering Science 62, 5854-5869.

Liu, Z., Zheng, Y., Jia, L., Zhang, Q., 2005. Study of bubble induced flow structure using PIV. Chemical Engineering Science 60, 3537-3552.

Mahinpey, N., Vejahati, F., Ellis, N., 2007. CFD simulation of gas-solid bubbling fluidized bed: an extensive assessment of drag models, Computational methods in Multiphase Flow IV. WIT Transactions on Engineering Sciences 56, 51-60.

McKeen, T., Pugsley, T., 2003. Simulation and experimental validation of a freely bubbling bed of FCC catalyst. Powder Technology 129, 139-152.

Min, J., Drake, J.B., Heindel, T.J., Fox, R.O., 2010. Experimental validation of CFD simulations of a lab-scale fluidized-bed reactor with and without side-gas infection. AIChE Journal 56, 1434-1446.

Nedderman, R.M., Laohakul, C., 1980. The thickness of the shear zone of flowing granular materials. Powder Technology 25, 91-100.

Otsu, N., 1979. A threshold selection method from gray-level histograms. IEEE Transactions on Systems, Man, and Cybernetics 9, 62-66.

Patil, D.J., van Sint Annaland, M., Kuipers, J.A.M., 2005. Critical comparison of hydrodynamic models for gas-solid fluidized beds-Part I: bubbling gas-solid fluidized beds operated with a jet. Chemical Engineering Science 60, 57-72.

Peirano, E., Delloume, V., Leckner, B., 2001. Two- or three-dimensional simulations of turbulent gas-solid flows applied to fluidization. Chemical Engineering Science 56, 4787-4799.

Raffel, M., Willert, C., Kompenhans, J., 2007. Particle Image Velocimetry, A Practical Guide. Springer, Berlin.

Sánchez-Delgado, S., Marugan-Cruz, C., Acosta-Iborra, A., Santana, D., 2010. Densephase velocity fluctuation in a 2-D fluidized bed. Powder Technology 200, 37-45.

Santana, D., Nauri, S., Acosta, A., García, N., Macías-Machín, A., 2005. Initial particle velocity spatial distribution from 2-D erupting bubbles in fluidized bed. Powder Technology 150, 1-8.
Schaeffer, D.G., 1987. Instability in the evolution equations describing incompressible granular flow. Journal of Differential Equations 66, 19-50.

Shen, L., Johnsson, F., Leckner, B., 2004. Digital image analysis of hydrodynamics two-dimensional bubbling fluidized beds. Chemical Engineering Science 59, 2607-2617.

Sveen J.K., 1998-2007. MATPIV. 〈http://www.math.uio.no/ jks/matpiv/〉.

Syamlal, M., O'Brien, T.J., 1987. Derivation of a drag coefficient from velocityvoidage correlation. U.S. Department of Energy, Office of Fossil; Energy National Energy Technology Laboratory, Morgantown, WV. Unpublished Report.

Syamlal, M., O'Brien, T.J., 1989. Computer simulation of bubbles in a fluidized bed. A.I.Ch.E. Symposium Series 85, 22-31.

Syamlal, M., Rogers, W., O'Brien T.J., 1993. MFIX Documentation: Vol. 1, Theory Guide. National Technical Information Service, Springfield, VA. DOE/METC9411004, NTIS/DE9400087.

Taghipour, F., Ellis, N., Wong, C., 2005. Experimental and computational study of gas-solid fluidized bed hydrodynamics. Chemical Engineering Science 60, 6857-6867.

Toei, R., Matsumo, R., 1967. The Coalescence of Bubbles in the Gas-Solid Fluidized Bed. Kyoto University, Japan.

Van der Hoef, M.A., van Sint Annaland, M., Kuipers, J.A.M., 2005. Computational fluid dynamics for dense gas-solid fluidized beds: a multiscale modeling strategy. China Particuology 3, 69-77.

Van Wachem, B.G.M., Almstedt, A.E., 2003. Methods for multiphase computational fluid dynamics. Chemical Engineering Journal 96, 81-98.

Van Wachem, B.G.M., Schouten, J.C., Krishna, R., van den Bleek, C.M., 1998 Computers Chemical Engineering 22, S299-S306.

Vejahati, F., Mahinpey, N., Ellis, N., Nikoo, M.B., 2009. CFD simulation of gas-solid bubbling fluidized bed: a new method for adjusting drag law. Canadian Journal Chemical Engineering 87, 19-30.

Wang, J., Liu, Y., 2010. EMMS-based Eulerian simulation on the hydrodynamics of a bubbling fluidized bed with FCC particles. Powder Technology 197, 241-246.

Wang, J., van der Hoef, M.A., Kuipers, J.A.M., 2010. Coarse grid simulation of bed expansion characteristics of industrial-scale gas-solid bubbling fluidized bed. Chemical Engineering Science 65, 2125-2131.

Yang, W.-C., 2003. Handbook of Fluidization and Fluid-Particle Systems. Marcel Dekker, New York. 